\title{
A Study of Weighted Polynomial Approximations for Orthogonal Polynomial Expansion
}

\author{
Ryozi Sakai \\ Department of Mathematics, Meijo University, Tenpaku-ku, Nagoya 468-8502, Japan \\ Email: ryozi@crest.ocn.ne.jp
}

\begin{abstract}
We investigate weighted polynomial approximations. Especially, we will study some facts related to the Fourier-type orthogonal expansion and the de la Vallée Poussin means. Then the estimate of the modulus of smoothness is important. To complete the theorems we need the Nikolskii-type inequality, higher order derivatives of approximation polynomials, the function with bounded variation, and others.
\end{abstract}

Keywords: Fourier-type orthogonal expansions, De la Vallée Poussin means, Modulus of smoothness.

\section{Introduction and Theorems}

In this paper, we study the weighted polynomial approximations. Especially, we treat of the partial sum of Fourier-type series and the de la Vallée Poussin means. Let $\mathbb{R}=(-\infty, \infty)$. We consider an exponential weight

$$
w(x)=\exp (-Q(x)), \quad x \in \mathbb{R},
$$

where $Q$ is an even and nonnegative function on $\mathbb{R}$. We assume that $w$ satisfies some conditions which will be stated in Section 2. We define a function $T=T_{w}$ by

$$
T(x):=\frac{x Q^{\prime}(x)}{Q(x)}, \quad x \neq 0 .
$$

We call $w$ a Freud-type weight if $T$ is bounded, and otherwise, $w$ is called an Erdös-type weight. For $\alpha>1, Q(x)=|x|^{\alpha}$ makes a typical example of Freud-type weights and $Q(x)=e^{x^{\alpha}}-1$ gives an Erdös-type weight.

Let $\left\{p_{n}\right\}$ be orthonormal polynomials with respect to a weight $w$, that is, $p_{n}$ is the polynomial of degree $n$ such that

$$
\int_{-\infty}^{\infty} p_{n}(x) p_{m}(x) w^{2}(x) \mathrm{d} x=\delta_{m n} \quad \text { (the Kronecker delta) }
$$

For $1 \leqslant p \leqslant \infty$, we denote the usual $L^{p}$ space on $\mathbb{R}$ by $L^{p}(\mathbb{R})$ (here for $p=\infty$, if $w f \in L^{\infty}(\mathbb{R})$, then we require $f$ to be continuous, and $w f$ to have limit 0 at $\pm \infty)$. For $w f \in L^{p}(\mathbb{R})$, we set

$$
s_{n}(f, x):=\sum_{k=0}^{n-1} b_{k}(f) p_{k}(x), \text { where } b_{k}(f)=\int_{-\infty}^{\infty} f(t) p_{k}(t) w^{2}(t) \mathrm{d} t
$$

for $n \in \mathbb{N}$ (the partial sum of Fourier-type series). The de la Vallée Poussin mean of order $n$ is defined by

$$
v_{n}(f, x):=\frac{1}{n} \sum_{j=n+1}^{2 n} s_{j}(f, x) .
$$

Up to date, we have obtained some results, such as our reports as follows. We define the MhaskarRakhmanov-Saff numbers $a_{n}$ as

$$
n=\frac{2}{\pi} \int_{0}^{1} \frac{a_{n} u Q^{\prime}\left(a_{n} u\right)}{\left(1-u^{2}\right)^{1 / 2}} \mathrm{~d} u .
$$


Let $1 \leqslant p \leqslant \infty$. For $w f \in L^{p}(\mathbb{R})$ and $n \in \mathbb{N}$, the degree of the weighted polynomial approximation $E_{p, n}(w ; f)$ is defined by

$$
E_{p, n}(w ; f):=\inf _{P \in \mathcal{P}_{n}}\|w(f-P)\|_{L_{p}(\mathbb{R})}
$$

where $\mathcal{P}_{n}$ is the class of all polynomials with degree $n$ at most. It is known that when $w \in \mathcal{F}\left(C^{2}+\right)$ and $w f \in L^{p}(\mathbb{R})$ (for $p=\infty$, we require $f$ to be continuous, and $w f$ to have limit 0 at $\pm \infty$ ), $E_{p, n}(w ; f$ ) converges 0 as $n \rightarrow \infty$ (see e.g. [3, Theorem 1.4]).

Result 1 ([9, Theorem 1, Corollary 14]). Let $w \in \mathcal{F}\left(C^{2}+\right)$ and let $w f \in L^{p}(\mathbb{R})$. Then, under the condition

$$
T\left(a_{n}\right) \leqslant c\left(\frac{n}{a_{n}}\right)^{2 / 3}
$$

there exists a constant $C>0$ such that

$$
\left\|\frac{w}{T^{1 / 4}} v_{n}(f)\right\|_{L^{p}(\mathbb{R})} \leqslant C\|w f\|_{L^{p}(\mathbb{R})},
$$

and

$$
\left\|w v_{n}(f)\right\|_{L^{p}(\mathbb{R})} \leqslant C\left\|T^{1 / 4} w f\right\|_{L^{p}(\mathbb{R})} .
$$

So,

$$
\left\|\frac{w}{T^{1 / 4}}\left(f-v_{n}(f)\right)\right\|_{L^{p}(\mathbb{R})} \leqslant C E_{p, n}(w, f),
$$

and

$$
\left\|w\left(f-v_{n}(f)\right)\right\|_{L^{p}(\mathbb{R})} \leqslant C E_{p, n}\left(T^{1 / 4} w, f\right) .
$$

Furthermore,

$$
\left\|w\left(f-v_{n}(f)\right)\right\|_{L^{p}(\mathbb{R})} \leqslant C T^{1 / 4}\left(a_{n}\right) E_{p, n}(w, f) .
$$

Moreover, if $f$ is absolutely continuous and $w f^{\prime} \in L^{p}(\mathbb{R})$, then

$$
\left\|\frac{w}{T^{1 / 4}} v_{n}(f)\right\|_{L^{p}(\mathbb{R})} \leqslant C \frac{a_{n}}{n}\left\|w f^{\prime}\right\|_{L^{p}(\mathbb{R})} .
$$

Result 2 ([10, Theorem 1.1]). Let $r \geqslant 2$ be an integer and let $w \in \mathcal{F}_{\lambda}\left(C^{4}+\right)(0<\lambda<(r+3) /(r+2))$ (see Section 2 for the definition), and let $1 \leqslant p \leqslant \infty$. Then there exists a constant $C>1$ such that if $1 \leqslant j \leqslant r$, and if $w f \in L^{p}(\mathbb{R})$, then

$$
\left\|\frac{w}{T^{(2 j+1) / 4}} v_{n}^{(j)}(f)\right\|_{L^{p}(\mathbb{R})} \leqslant C\left(\frac{n}{a_{n}}\right)^{j}\|w f\|_{L^{p}(\mathbb{R})}
$$

holds for all $n \in \mathbb{N}$.

Result 3 ([11, Theorem 1.1]). Let $w \in \mathcal{F}_{\lambda}\left(C^{3}+\right)$ with $0<\lambda<3 / 2$. Suppose that $f$ is continuous and has a bounded variation on any compact interval of $\mathbb{R}$. If $f$ satisfies

$$
\int_{-\infty}^{\infty} w(x)|\mathrm{d} f(x)|<\infty
$$

then

$$
\lim _{n \rightarrow \infty}\left\|\frac{w}{T^{1 / 4}}\left(f-s_{n}(f)\right)\right\|_{L^{\infty}(\mathbb{R})}=0 .
$$

The completion of theorems with the weight $w \in \mathcal{F}_{\lambda}\left(C^{m}+\right)$ is guaranteed by the following theorems; 
Result 4 ([8, Theorem 4.1,4.2 and (4.11)]). Let $0<\lambda<3 / 2$ and $\alpha \in \mathbb{R}$. Then for $w=\exp (-Q) \in$ $\mathcal{F}_{\lambda}\left(C^{3}+\right)$, we can construct a new weight $w_{\alpha} \in \mathcal{F}\left(C^{2}+\right)$ such that

$$
T(x)^{\alpha} w(x) \sim w_{\alpha}(x), \quad x \in \mathbb{R}
$$

and the following holds:

$$
a_{n}\left(w_{\alpha}\right) \sim a_{n}=a_{n}(w), \quad n=1,2,3, \ldots .
$$

In fact, there exists $c>1$ such that

$$
a_{n / c}\left(w_{\alpha}\right) \leqslant a_{n}=a_{n}(w) \leqslant a_{c n}\left(w_{\alpha}\right), \quad n=1,2,3, \ldots
$$

and

$$
T_{w_{\alpha}}(x) \sim T(x)=T_{w}(x) \quad x \in \mathbb{R} .
$$

Let $w=\exp (-Q) \in F_{\lambda}\left(C^{m+1}+\right)$ for an integer $m>0$, and let $\alpha=\alpha_{1}+\alpha_{2}+\ldots+\alpha_{m}$. Then inductively we construct new weights $w_{\alpha_{1}},\left(w_{\alpha_{1}}\right)_{\alpha_{2}}, \ldots$.

When we consider the function with higher order derivatives, we need the following important theorem;

Result 5 ([7, Corollary 8]). Let $w \in \mathcal{F}\left(C^{2}+\right)$, and let $r \geqslant 0$ be an integer. Let $1 \leqslant p \leqslant \infty$, and let $w f^{(r)} \in L_{p}(\mathbb{R})$. Then we have

$$
E_{p, n}(f, w) \leqslant C\left(\frac{a_{n}}{n}\right)^{k}\left\|w f^{(k)}\right\|_{L_{p}(\mathbb{R})}, \quad k=1,2, \ldots, r,
$$

and equivalently,

$$
E_{p, n}(f, w) \leqslant C\left(\frac{a_{n}}{n}\right)^{k} E_{p, n-k}\left(f^{(k)}, w\right) .
$$

In this paper our purpose is to improve Results 1-3. Now, we can give some new results as below. To prove them we need many lemmas. Avoiding complex representations we will give proofs of lemmas in Appendix. We start from the estimate of the modulus of smoothness.

Theorem 1. Let $w=\exp (-Q) \in \mathcal{F}_{\lambda}\left(C^{r+1}+\right)(0<\lambda<(r+1) / r)$, where $r$ is a positive integer, and let $1 \leqslant p \leqslant \infty$. Let $f^{(r-1)}(x)$ be absolutely continuous and $\left\|w f^{(r)}\right\|_{L^{p}(\mathbb{R})}<\infty$, then we have

$$
\tilde{\omega}_{r, p}(f, w, t) \leqslant C t^{r}\left\|w f^{(r)}\right\|_{L^{p}(\mathbb{R})} .
$$

Theorem 2. Let $w \in \mathcal{F}_{\lambda}\left(C^{3}+\right), 0<\lambda<3 / 2$, and let $1 \leqslant p \leqslant \infty$. Suppose that for a positive integer $r$, $f^{(r-1)}(x)$ is continuous on $\mathbb{R}$ and has a bounded variation on any compact interval of $\mathbb{R}$. Furthermore $f^{(r-1)}(x)$ satisfies

$$
\int_{-\infty}^{\infty} w(x)\left|\mathrm{d} f^{(r-1)}(x)\right|<\infty
$$

then we have

$$
\tilde{\omega}_{r, p}(f, w, t) \leqslant C t^{r-1+1 / p} o(1)^{1-1 / p}\left\{\int_{\mathbb{R}} w(y)\left|\mathrm{d} f^{(r-1)}\right|\right\}^{1 / p},
$$

where $\tilde{w}_{r, p}(f, w . t)$ is defined in Section 3.

Theorem 3. Let $w \in \mathcal{F}\left(C^{2}+\right)$, and let (1.2) be satisfied. Let $1 \leqslant p \leqslant \infty$. Suppose that $f$ is continuous and has a bounded variation on any compact interval of $\mathbb{R}$. If $f$ satisfies (1.6), then we have

$$
\left\|\frac{w}{T^{1 / 4}}\left(f-v_{n}(f)\right)\right\|_{L^{p}(\mathbb{R})} \leqslant C o_{n}(1)^{1-1 / p}\left(\frac{a_{n}}{n}\right)^{\frac{1}{p}},
$$

where $C>0$ is a constant and

$$
o_{n}(1):=\tilde{\omega}_{1, \infty}\left(f, w, L \frac{a_{n}}{n}\right) \rightarrow 0 \text { as } n \rightarrow \infty .
$$


Now, we treat of an absolutely continuous function.

Theorem 4. Let $w \in \mathcal{F}\left(C^{2}+\right)$, and let (1.2) be satisfied. Let $1 \leqslant p \leqslant \infty$, and let $r$ be a positive integer. Suppose that $f^{(r-1)}(x)$ is absolutely continuous and $w f^{(r)} \in L^{p}(\mathbb{R})$, then we have

$$
\left\|\frac{w}{T^{1 / 4}}\left(f-v_{n}(f)\right)\right\|_{L^{p}(\mathbb{R})} \leqslant C\left(\frac{a_{n}}{n}\right)^{r}\left\|w f^{(r)}\right\|_{L^{p} \mathbb{R}} .
$$

Theorem 5. Let $w=\exp (-Q) \in \mathcal{F}_{\lambda}\left(C^{r+2}+\right), 0<\lambda<(r+2) /(r+1)$, where $1 \leqslant r$ is an integer, and let (1.2) be satisfied. Let $1 \leqslant p \leqslant \infty$. We suppose that $f^{(r-1)}$ is absolutely continuous. Then for $T^{1 / 4} w f^{(r)} \in L^{p}(\mathbb{R})$ we have

$$
\left\|\frac{w}{T^{i / 2}}\left(f^{(i)}-v_{n}^{(i)}\right)\right\|_{L^{p}(\mathbb{R})} \leqslant C\left(\frac{a_{n}}{n}\right)^{r-i} E_{p, n-r}\left(T^{1 / 4} w ; f^{(r)}\right),
$$

and for $w f^{(r)} \in L^{P}(\mathbb{R})$ we see

$$
\left\|\frac{w}{T^{(2 i+1) / 4}}\left(f^{(i)}-v_{n}^{(i)}\right)\right\|_{L^{p}(\mathbb{R})} \leqslant C\left(\frac{a_{n}}{n}\right)^{r-i} E_{p, n-r}\left(w ; f^{(r)}\right) .
$$

Furthermore, for $T^{(2 i+1) / 4} w f^{(r)} \in L^{p}(\mathbb{R})$ we have

$$
\left\|w\left(f^{(i)}-v_{n}^{(i)}\right)\right\|_{L^{p}(\mathbb{R})} \leqslant C\left(\frac{a_{n}}{n}\right)^{r-i} E_{p, n-r}\left(T^{(2 i+1) / 4} w ; f^{(r)}\right) .
$$

Especially, for the Freud-type weight w we have

$$
\left\|w\left(f^{(i)}-v_{n}^{(i)}\right)\right\|_{L^{p}(\mathbb{R})} \leqslant C\left(\frac{a_{n}}{n}\right)^{r-i} E_{p, n-r}\left(w ; f^{(r)}\right) .
$$

The following theorem is an extension of Theorem 3.

Theorem 6. Let $w=\exp (-Q) \in \mathcal{F}_{\lambda}\left(C^{r+2}+\right), 0<\lambda<(r+2) /(r+1)$, where $1 \leqslant r$ is an integer, and $1 \leqslant p \leqslant \infty$. Suppose that $f^{(r-1)}(x)$ is continuous on $\mathbb{R}$ and has a bounded variation on any compact interval of $\mathbb{R}$, and (1.8) is satisfied. Let $1 \leqslant p \leqslant \infty$. Then we have

$$
\begin{gathered}
\left\|\frac{w}{T^{(2 i+1) / 4}}\left(f^{(i)}-v_{n}^{(i)}\right)\right\|_{L^{p}(\mathbb{R})} \leqslant C\left(\frac{a_{n}}{n}\right)^{r-i-1+1 / p} O_{n}(1)^{1-1 / p}\left\{\int_{\mathbb{R}} w(y)\left|\mathrm{d} f^{(r-1)}\right|\right\}^{1 / p}, \\
i=0,1, \ldots, r-1 .
\end{gathered}
$$

Theorem 7. Let $w \in \mathcal{F}_{\lambda}\left(C^{3}+\right), 0<\lambda<3 / 2$. Suppose that $f$ is absolutely continuous and $w f^{\prime} \in$ $L^{p}(\mathbb{R}) \cap L^{2}(\mathbb{R}) \quad(1 \leqslant p \leqslant \infty)$. Then we have

$$
\left\|\frac{w}{T^{1 / 4}}\left(f-s_{n}\right)\right\|_{L^{p}(\mathbb{R})} \leqslant C_{f}\left(\frac{a_{n}}{n}\right)^{\frac{1}{2}+\frac{1}{p}} \begin{cases}n^{\frac{1}{p}-\frac{1}{2}}, & 1 \leqslant p<2 \\ 1, & 2 \leqslant p \leqslant \infty\end{cases}
$$

where $C_{f}:=C\left\{\left\|w f^{\prime}\right\|_{L^{p}(\mathbb{R})}+\left\|w f^{\prime}\right\|_{L^{2}(\mathbb{R})}\right\}$.

In this theorem we see that the best estimation is given for $p=2$.

Theorem 7 is extended as follows.

Theorem 8. Let $w \in \mathcal{F}_{\lambda}\left(C^{r+2}+\right), 0<\lambda<(r+2) /(r+1)$, where $r$ is a positive integer, and $1 \leqslant p \leqslant \infty$. Suppose that $f^{(r-1)}(x)$ is continuous on $\mathbb{R}$ and has a bounded variation on any compact interval of $\mathbb{R}$, and (1.8) is satisfied. Then we have

$$
\begin{aligned}
& \left\|\frac{w}{T^{(2 i+1) / 4}}\left(f^{(i)}-s_{n}^{(i)}\right)\right\|_{L^{p}(\mathbb{R})} \\
& \leqslant C\left(\frac{a_{n}}{n}\right)^{r-i-1+\frac{1}{p}}\left\{\int_{\mathbb{R}} w(y)\left|\mathrm{d} f^{(r-1)}\right|\right\}^{1 / p}\left\{\begin{array}{lr}
o(1)^{1-1 / p} n^{\frac{1}{p}-\frac{1}{2}}, & 1 \leqslant p<2 ; \\
o(1)^{\frac{1}{2}}, & 2 \leqslant p \leqslant \infty .
\end{array}\right. \\
& i=0,1, \ldots, r-1,
\end{aligned}
$$

where $C_{f}$ is a constant depending only on $f$ and $\delta_{n}$ is defined by (1.10). 
In Section 2, we give definitions of $\mathcal{F}\left(C^{2}+\right)$ and $\mathcal{F}_{\lambda}\left(C^{m+2}+\right)$. They are classes of exponential weights which are treated in this paper. Some related notations are also given. In Section 3 we give the estimate of modulus of smoothness. In Section 4 we give estimates of the de la Vallée Poussin means. In Section 5 we give estimates for the partial sum of Fourier-type expansion. To clarify our main theorems the proofs of other results are stated in Appendix.

For any nonzero real valued functions $f(x)$ and $g(x)$, if there exist constants $C_{1}, C_{2}>0$ independent of $x$ such that $C_{1} g(x) \leqslant f(x) \leqslant C_{2} g(x)$ for all $x$ in the range, then we write $f(x) \sim g(x)$. Similarly, for any two sequences of positive numbers $\left\{c_{n}\right\}_{n=1}^{\infty}$ and $\left\{d_{n}\right\}_{=1}^{\infty}$ we define $c_{n} \sim d_{n}$.

Throughout this paper, $C, C_{1}, C_{2}, \ldots$ denote positive constants independent of $n, x, t$ or polynomials $P_{n}(x)$. The same symbol does not necessarily denote the same constant in different occurrences.

\section{Definitions of Classes $\mathcal{F}\left(C^{2}+\right), \mathcal{F}_{\lambda}\left(C^{m+2}+\right)$, and Notations}

We say that an exponential weight $w=\exp (-Q)$ belongs to a class $\mathcal{F}\left(C^{2}+\right)$, when $Q: \mathbb{R} \rightarrow[0, \infty)$ is a continuous and even function and satisfies the following conditions:

(a) $Q^{\prime}(x)$ is continuous on $\mathbb{R}$ and $Q(0)=0$.

(b) $Q^{\prime \prime}(x)$ exists and is positive on $\mathbb{R} \backslash\{0\}$.

(c) $\lim _{x \rightarrow \infty} Q(x)=\infty$.

(d) The function

$$
T(x):=\frac{x Q^{\prime}(x)}{Q(x)}, \quad x \neq 0
$$

is quasi-increasing in $(0, \infty)$ (i.e. there exists $c \geqslant 1$ such that $T(x) \leqslant c T(y)$ whenever $0<x<y)$, and there exists $\Lambda>1$ such that

$$
T(x) \geqslant \Lambda, \quad x \in \mathbb{R} \backslash\{0\} .
$$

From now on we set $T(0)=\Lambda$. Then $T(x) \geqslant \Lambda$ on $\mathbb{R}$

(e) There exists $C>1$ such that

$$
\frac{Q^{\prime \prime}(x)}{\left|Q^{\prime}(x)\right|} \leqslant C_{1} \frac{\left|Q^{\prime}(x)\right|}{Q(x)}, \text { a.e. } x \in \mathbb{R}
$$

and

$$
C \frac{Q^{\prime \prime}(x)}{\left|Q^{\prime}(x)\right|} \geqslant \frac{\left|Q^{\prime}(x)\right|}{Q(x)}, \text { a.e. } x \in \mathbb{R} \backslash J \text {. }
$$

Let $w \in \mathcal{F}\left(C^{2}+\right)$, and let $\lambda>0$. We write $w \in \mathcal{F}_{\lambda}\left(C^{2}+\right)$, if there exist $K>1$ and $C>1$ such that for all $|x| \geqslant K$,

$$
\frac{\left|Q^{\prime}(x)\right|}{Q(x)^{\lambda}} \leqslant C
$$

holds. We also write $w \in \mathcal{F}_{\lambda}\left(C^{m+2}+\right)$ for a nonnegative integer, if $w \in \mathcal{F}_{\lambda}\left(C^{2}+\right), Q \in C^{m+2}(\mathbb{R} \backslash\{0\})$ and

$$
\left|\frac{Q^{\prime \prime}(x)}{Q^{\prime}(x)}\right| \sim\left|\frac{Q^{(k+1)}(x)}{Q^{(k)}(x)}\right|(k=2, \ldots, m), \quad\left|\frac{Q^{(m+2)}(x)}{Q^{(m+1)}(x)}\right| \leqslant C\left|\frac{Q^{(m+1)}(x)}{Q^{(m)}(x)}\right|
$$

hold for every $|x| \geqslant K$. Clearly $\mathcal{F}_{\lambda}\left(C^{m+2}+\right) \subset \mathcal{F}_{\lambda}\left(C^{2}+\right) \subset \mathcal{F}\left(C^{2}+\right)$.

Let $u \geqslant 0, \alpha>0$ with $\alpha+u>1$ and let $l \in \mathbb{N}$. We set

$$
Q(x)=|x|^{u} Q_{l, \alpha}(x)=\exp _{l}\left(|x|^{\alpha}\right)-\exp _{l}(0),
$$

where $\exp _{l}(x)=\exp (\exp (\exp \ldots \exp x) \ldots) \quad(l$ times $)$. 
Set

$$
\sigma(t):=\inf \left\{a_{u} ;\left(a_{u} / u\right) \leqslant t\right\}, \quad t>0 .
$$

We see that $a_{u} / u$ is monotonically decreasing for every $t>0$, so there is a unique $u>0$ such that

$$
t=\frac{a_{u}}{u} \text { and } \sigma(t)=a_{u}
$$

and hence $\lim _{t \rightarrow+0} \sigma(t)=\infty$. Furthermore we have

$$
a_{n} \leqslant a_{c_{0} n} \leqslant c_{0} a_{n}
$$

for every $c_{0} \geqslant 1$ and every $n \in \mathbb{N}$.

Lastly we recall the $r$-th order of the modulus of smoothness $\omega_{r, p}(w ; f ; t)$, which is defined as follows (cf. [1]): Let $r$ be a positive integer, and let $1 \leqslant p \leqslant \infty$. We set

$$
\Delta_{h}^{r}(f, x):=\sum_{i=0}^{r}\left(\begin{array}{r}
r \\
i
\end{array}\right)(-1)^{i} f\left(x+\frac{r h}{2}-i h\right), \quad x \in \mathbb{R} .
$$

For the Freud-type weight,

$$
\omega_{r, p}(f, w, t):=\sup _{0<h \leqslant t}\left\|w \Delta_{h}^{r}(f, x)\right\|_{L^{p}(|x| \leqslant \sigma(2 t))}+\inf _{P \in \mathcal{P}_{r-1}}\|(f-P) w\|_{L^{p}(|x| \geqslant \sigma(4 t))} .
$$

For the Erdös-type weight,

$$
\omega_{r, p}(f, w, t):=\sup _{0<h \leqslant t}\left\|w \Delta_{h \Phi_{t}(x)}^{r}(f, x)\right\|_{L^{p}(|x| \leqslant \sigma(2 t))}+\inf _{P \in \mathcal{P}_{r-1}}\|(f-P) w\|_{L^{p}(|x| \geqslant \sigma(4 t))},
$$

where

$$
\Phi_{t}(x):=\sqrt{\left|1-\frac{|x|}{\sigma(t)}\right|}+\frac{1}{\sqrt{T(\sigma(t))}} .
$$

We remark that if $T(x)$ is bounded, then we see $\Phi_{t}(x) \sim 1$. So, (2.2) means (2.1). Hence, in the rest of this paper we consider only (2.2). Unfortunately, $\omega_{r, p}(f, w, t)$ is inconvenient for our use, so we will modify it. For an integer $r \geqslant 1$, let $f^{(r-1)}$ be continuous. Then we define the modulus of smoothness $\tilde{\omega}_{r, p}$ as follows.

$$
\tilde{\omega}_{r, p}(f, w, t):=\sup _{0<h \leqslant t}\left\|w \Delta_{h \Phi_{t}(x)}^{r}(f, x)\right\|_{L^{p}(|x| \leqslant \sigma(2 t))}+\left\|w\left(f-P_{r-1, f}\right)\right\|_{L^{p}(|x| \geqslant \sigma(4 t))} .
$$

Here, we set $P_{r-1, f} \in \mathcal{P}_{r-1}$ such as

$$
P_{r-1, f}^{(j)}(0)=f^{(j)}(0), \quad j=0,1, \ldots, r-1 .
$$

Clearly, we see $\omega_{r, p}(f, w, t) \leqslant \tilde{\omega}_{r, p}(f, w, t)$.

\section{Estimate of Modulus of Smoothness}

In this section we give the proofs of Theorems 1,2. To do them we need some lemmas.

Theorem $9\left(\left[A_{1}\right]\right)$. Let $w=\exp (-Q) \in \mathcal{F}\left(C^{2}+\right)$.

(a) We have

$$
\left|Q^{\prime}(x) w(x) \int_{0}^{x} w^{-1}(t) \mathrm{d} t\right| \leqslant C, \quad x \geqslant 0
$$


(b) Let $1 \leqslant p \leqslant \infty$. If $g$ is absolutely continuous, $g(0)=0$ and $g^{\prime} \in L_{w}^{p}(\mathbb{R})$, then for any fixed constant $\gamma \geqslant 0$,

$$
\left\|Q^{\prime} w g\right\|_{L^{p}(|x| \geqslant \gamma)} \leqslant C\left\|w g^{\prime}\right\|_{L^{p}(\mathbb{R})} .
$$

Furthermore, when $w=\exp (-Q) \in \mathcal{F}_{\lambda}\left(C^{r+1}+\right) \subset \mathcal{F}\left(C^{2}+\right)(0<\lambda<(r+1) / r)$, where $r$ is a positive integer $)$, further $g^{(r-1)}$ is absolutely continuous, $g^{(j)}(0)=0, \quad j=0,1, \ldots, r-1$ and $w^{(r)} \in L^{p}(\mathbb{R})$, we see

$$
\left\|\left(Q^{\prime}\right)^{r} w g\right\|_{L^{p}(|x| \geqslant \gamma)} \leqslant C\left\|w g^{(r)}\right\|_{L^{p}(\mathbb{R})} .
$$

From (3.3) we see that $\left\|w g^{(r)}\right\|_{L^{p}(\mathbb{R})}<\infty$ means $\left\|w g^{(i)}\right\|_{L^{p}(\mathbb{R})}<\infty, \quad i=0,1, \ldots, r-1$.

The proof of Theorem 9 is given in Appendix. We denote this fact by $\left[A_{1}\right]$. As we see below we will use the same notation similarly, that is, $P\left(\left[A_{i}\right]\right)$ means that the proof of $P$ is given in Appendix $\left[A_{i}\right]$.

The modulus of smoothness $\tilde{\omega}_{r, p}$ in $(2.3)$ is very useful for the approximation theory.

Proof of Theorem 1. We use Hölder's inequality. Let $1 / p+1 / q=1,1 \leqslant p \leqslant \infty$. We see

$$
\begin{aligned}
& \left\|w \Delta_{h \Phi_{t}(x)}^{r}(f, x)\right\|_{L^{p}(|x| \leqslant \sigma(2 t))}^{p} \\
& =\int_{|x| \leqslant \sigma(2 t)}\left|w(x) \int_{-h \Phi_{t}(x) / 2}^{h \Phi_{t}(x) / 2} \ldots \int_{-h \Phi_{t}(x) / 2}^{h \Phi_{t}(x) / 2} f^{(r)}\left(x+t_{1}+\ldots+t_{r}\right) \mathrm{d} t_{1} \ldots \mathrm{d} t_{r}\right|^{p} \mathrm{~d} x \\
& \leqslant C \int_{|x| \leqslant \sigma(2 t)}\left|\int_{-h \Phi_{t}(x) / 2}^{h \Phi_{t}(x) / 2} \ldots \int_{-h \Phi_{t}(x) / 2}^{h \Phi_{t}(x) / 2} w\left(x+t_{1}+\ldots+t_{r}\right) f^{(r)}\left(x+t_{1}+\ldots+t_{r}\right) \mathrm{d} t_{1} \ldots \mathrm{d} t_{r}\right|^{p} \mathrm{~d} x \\
& \leqslant C \int_{|x| \leqslant \sigma(2 t)}\left|\int_{-h}^{h} \ldots \int_{-h}^{h} w\left(x+t_{1}+\ldots+t_{r}\right) f^{(r)}\left(x+t_{1}+\ldots+t_{r}\right) \mathrm{d} t_{1} \ldots \mathrm{d} t_{r}\right|^{p} \mathrm{~d} x \\
& \leqslant C \int_{|x| \leqslant \sigma(2 t)}\left\{\int_{-h}^{h} 1^{q} \mathrm{~d} t_{r}\right\}^{p / q} \\
& \times\left\{\int_{-h}^{h}\left|\int_{-h}^{h} \ldots \int_{-h}^{h} w\left(x+t_{1}+\ldots+t_{r}\right) f^{(r)}\left(x+t_{1}+\ldots+t_{r}\right) \mathrm{d} t_{1} \ldots \mathrm{d} t_{r-1}\right|^{p} \mathrm{~d} t_{r}\right\} \mathrm{d} x \\
& \leqslant C \int_{|x| \leqslant \sigma(2 t)}\left\{\int_{-h}^{h} 1^{q} \mathrm{~d} t_{r}\right\}^{p / q}\left\{\int_{-h}^{h} 1^{q} \mathrm{~d} t_{r-1}\right\}^{p / q} \\
& \times\left\{\int_{-h}^{h} \int_{-h}^{h}\left|\int_{-h}^{h} \ldots \int_{-h}^{h} w\left(x+t_{1}+\ldots+t_{r}\right) f^{(r)}\left(x+t_{1}+\ldots+t_{r}\right) \mathrm{d} t_{1} \ldots \mathrm{d} t_{r-2}\right|^{p}\right. \\
& \left.\times \mathrm{d} t_{r} \mathrm{~d} t_{r-1}\right\} \mathrm{d} x \\
& \leqslant C \int_{|x| \leqslant \sigma(2 t)}\left\{\int_{-h}^{h} 1^{q} \mathrm{~d} t_{r}\right\}^{p / q}\left\{\int_{-h}^{h} 1^{q} \mathrm{~d} t_{r}\right\}^{p / q} \ldots .\left\{\int_{-h}^{h} 1^{q} \mathrm{~d} t_{r}\right\}^{p / q} \\
& \times\left\{\int_{-h}^{h} \int_{-h}^{h} \ldots \int_{-h}^{h}\left|w\left(x+t_{1}+\ldots+t_{r}\right) f^{(r)}\left(x+t_{1}+\ldots+t_{r}\right)\right|^{p} \mathrm{~d} t_{r} \mathrm{~d} t_{r-1} \ldots \mathrm{d} t_{1}\right\} \mathrm{d} x \\
& \leqslant C\left\{\int_{-h}^{h} 1^{q} \mathrm{~d} t_{r}\right\}^{p / q}\left\{\int_{-h}^{h} 1^{q} \mathrm{~d} t_{r}\right\}^{p / q} \ldots .\left\{\int_{-h}^{h} 1^{q} \mathrm{~d} t_{r}\right\}^{p / q} \\
& \times\left\{\int_{-h}^{h} \int_{-h}^{h} \ldots \int_{-h}^{h} \int_{\mathbb{R}}\left|w\left(x+t_{1}+\ldots+t_{r}\right) f^{(r)}\left(x+t_{1}+\ldots+t_{r}\right)\right|^{p} \mathrm{~d} x \mathrm{~d} t_{r} \mathrm{~d} t_{r-1} \ldots \mathrm{d} t_{1}\right\} \\
& \leqslant C t^{p r / q} t^{r}\left\|w f^{(r)}\right\|_{L^{p}(\mathbb{R})}^{p} \text {. }
\end{aligned}
$$

Therefore, we have

$$
\left\|w \Delta_{h \Phi_{t}(x)}^{r}(f, x)\right\|_{L^{\infty}(|x| \leqslant \sigma(2 t))} \leqslant C t^{r / q} t^{r / p}\left\|w f^{(r)}\right\|_{L^{p}(\mathbb{R})}=C t^{r}\left\|w f^{(r)}\right\|_{L^{p}(\mathbb{R})}
$$


Next, by Theorem 9 with $\gamma=\sigma(4 t)$ we have

$$
\left.\left\|\left(Q^{\prime}(x)\right)^{r} w(x)\left(f(x)-P_{r-1, f}(x)\right)\right\|_{L^{p}(|x| \geqslant \sigma(4 t))} \leqslant C \| w(x) f^{(r)}(x)\right) \|_{L^{p}(\mathbb{R})}<\infty,
$$

where the polynomial $P_{r-1, f}$ is defined by (2.4). Therefore, we see

$$
\begin{aligned}
& \left\|w(x)\left(f(x)-P_{r-1, f}(x)\right)\right\|_{L^{p}(|x| \geqslant \sigma(4 t))} \leqslant\left(\frac{1}{Q^{\prime}(\sigma(4 t))}\right)^{r}\left\|w f^{(r)}\right\|_{L^{p}(\mathbb{R})} \\
& \leqslant C t^{r}\left\|w f^{(r)}\right\|_{L^{p}(\mathbb{R})} .
\end{aligned}
$$

Consequently, by $\omega_{r, p}(f, w, t) \leqslant \tilde{\omega}_{r, p}(f, w, t)$ we have the result $(1.7)$.

From the condition (1.8) with $r=1$ and $g$ we obtain the following facts.

Lemma 10 (cf. [11, Lemma 3.3]). Let $w \in \mathcal{F}\left(C^{2}+\right)$. Suppose that $g$ is continuous on $\mathbb{R}$ and has a bounded variation on any compact interval of $\mathbb{R}$, and $\int_{-\infty}^{\infty} w(x)|\mathrm{d} g(x)|<\infty$ holds. Then wg $\in L^{1}(\mathbb{R})$ and $w g$ has a limit 0 at $\pm \infty$.

Proof. Let $w(x)=\exp (-Q(x))$. We may suppose $g(0)=0$. Since $Q^{\prime}(x)$ is nondecreasing in $(0, \infty)$, Fubini's Theorem gives us

$$
\begin{aligned}
& \int_{1}^{\infty}|g(x) w(x)| \mathrm{d} x \leqslant \frac{1}{Q^{\prime}(1)} \int_{1}^{\infty} Q^{\prime}(x)|g(x)| w(x) \mathrm{d} x \\
& =\frac{1}{Q^{\prime}(1)} \int_{1}^{\infty} Q^{\prime}(x)\left|\int_{0}^{x} \mathrm{~d} g(t)\right| w(x) \mathrm{d} x \leqslant \frac{1}{Q^{\prime}(1)} \int_{0}^{\infty} Q^{\prime}(x)\left(\int_{0}^{x}|\mathrm{~d} g(t)|\right) w(x) \mathrm{d} x \\
& =\frac{1}{Q^{\prime}(1)} \int_{0}^{\infty}\left(\int_{t}^{\infty} Q^{\prime}(x) w(x) \mathrm{d} x\right)|\mathrm{d} g(t)|=\frac{1}{Q^{\prime}(1)} \int_{0}^{\infty} w(t)|\mathrm{d} g(t)|<\infty .
\end{aligned}
$$

Similarly $\int_{-\infty}^{-1}|g(x) w(x)| \mathrm{d} x<\infty$ and clearly $\int_{-1}^{1}|g(x) w(x)| \mathrm{d} x<\infty, g w \in L^{1}(\mathbb{R})$ follows. For any $\varepsilon>0$, we take $R>0$ such that $\int_{R}^{\infty} w(t)|\mathrm{d} g(t)|<\varepsilon$. If $R<x<\infty$, then we have

$$
|g(x)-g(R)|=\left|\int_{R}^{x} \mathrm{~d} g(t)\right| \leqslant \frac{1}{w(x)} \int_{R}^{x} w(t)|\mathrm{d} g(t)| \leqslant \frac{\varepsilon}{w(x)},
$$

which implies $\lim _{x \rightarrow \infty} g(x) w(x)=0$. Similarly $g(x) w(x) \rightarrow 0$ as $x \rightarrow-\infty$. In particular, $g w \in L^{\infty}(\mathbb{R})$.

To prove Theorem 2 we need the following lemmas. The proofs of them will be given in Appendix.

Theorem $11\left(\left[A_{2}\right]\right.$, cf. $\left.[11,(3.2)]\right)$. Let $w \in \mathcal{F}_{\lambda}\left(C^{3}+\right)(0<\lambda<3 / 2)$, and let $1 \leqslant p \leqslant \infty$. Suppose $w f \in L^{1}(\mathbb{R}) \cap L^{\infty}(\mathbb{R})$. Then we have

$$
\tilde{\omega}_{r, p}(f, w, t) \leqslant \tilde{\omega}_{r, \infty}^{1-1 / p}(f, w, t) \tilde{\omega}_{r, 1}^{1 / p}(f, w, t) .
$$

Theorem 12 ([A $\left.A_{3}\right]$, cf. $\left[2,11\right.$ (Proposition 3.2)]). Let $w \in \mathcal{F}_{\lambda}\left(C^{3}+\right), 0<\lambda<3 / 2$. Suppose that for a positive integer $r, f^{(r-1)}(x)$ is continuous on $\mathbb{R}$ and has a bounded variation on any compact interval of $\mathbb{R}$. Furthermore $f^{(r-1)}(x)$ satisfies (1.8). Then we have

$$
\tilde{\omega}_{r, 1}(w, f, t) \leqslant C t^{r} \int_{\mathbb{R}} w(y)\left|\mathrm{d} f^{(r-1)}\right|,
$$

and

$$
\tilde{\omega}_{r, \infty}(w, f, t) \leqslant C t^{r-1} o(1) .
$$

Proof of Theorem 2. It immediately follows from Theorems 11 and 12 (note Lemma 10). 


\section{Estimates of the De La Vallée Poussin Means}

We need a Jackson-type theorem.

Lemma $13([1,11(3.3)])$. Let $w \in \mathcal{F}\left(C^{2}+\right)$, and let $0<p \leqslant \infty$. We suppose $w f \in L_{p}(\mathbb{R})($ for $p=\infty$, we require $f$ to be continuous, and $w f$ to vanish at $\pm \infty)$. Then there exists a constant $C>0$ such that for $n \geqslant C_{1}$,

$$
E_{p, n}(f, w) \leqslant C \omega_{r, p}\left(f, w, L \frac{a_{n}}{n}\right) \leqslant C \tilde{\omega}_{r, p}\left(f, w, L \frac{a_{n}}{n}\right),
$$

where $C_{1}, L>0$ do not depend on $f$ and $n$.

Now, the proof of Theorem 3 is simple.

Proof of Theorem 3. By Result 1(1.3) we have

$$
\left\|\frac{w}{T^{1 / 4}}\left(f-v_{n}(f)\right)\right\|_{L_{p}(\mathbb{R})} \leqslant C E_{p, n}(w, f) \leqslant C \tilde{\omega}_{1, p}\left(f, w, L \frac{a_{n}}{n}\right) .
$$

Now using Theorem 2 with $r=1$, we have the result.

The proof of Theorem 4 is also simple.

Proof of Theorem 4. By by Result 1 (1.3) we have

$$
\left\|\frac{w}{T^{1 / 4}}\left(f-v_{n}(f)\right)\right\|_{L_{p}(\mathbb{R})} \leqslant C E_{p, n}(w, f) .
$$

Now from Result 5,

$$
\left\|\frac{w}{T^{1 / 4}}\left(f-v_{n}(f)\right)\right\|_{L_{p}(\mathbb{R})} \leqslant C\left(\frac{a_{n}}{n}\right)^{r}\left\|w f^{(r)}\right\|_{L^{p}(\mathbb{R})}
$$

To prove Theorem 5 we need the following lemmas.

Theorem $14\left(\left[A_{4}\right]\right)$. Let $w=\exp (-Q) \in \mathcal{F}_{\lambda}\left(C^{r+2}+\right), 0<\lambda<(r+2) /(r+1)$, where $1 \leqslant r$ is an integer, and let (1.2) be satisfied. Let $1 \leqslant p \leqslant \infty$. We suppose that an absolutely continuous function $f^{(r-1)}$ satisfies $T^{1 / 4} w f^{(r)} \in L^{p}(\mathbb{R})$. Then there exists a constant $C \geqslant 1$ such that if

$$
\|w(f-P)\|_{L^{p}(\mathbb{R})} \leqslant \varepsilon
$$

holds for some $n>r, P \in \mathcal{P}_{2 n}$ and $\varepsilon>0$, then

$$
\left\|\frac{w}{T^{i / 2}}\left(f^{(i)}-P^{(i)}\right)\right\|_{L^{p}(\mathbb{R})} \leqslant C\left\{E_{p, n-i}\left(T^{1 / 4} w ; f^{(i)}\right)+\left(\frac{n}{a_{n}}\right)^{i} \varepsilon\right\}
$$

holds for all $i=1,2, \ldots, r$. In particular, if

$$
\|w(f-P)\|_{L^{p}(\mathbb{R})} \leqslant C E_{p, n}\left(T^{1 / 4} w ; f\right),
$$

then

$$
\left\|\frac{w}{T^{i / 2}}\left(f^{(i)}-P^{(i)}\right)\right\|_{L^{p}(\mathbb{R})} \leqslant C\left(\frac{a_{n}}{n}\right)^{r-i} E_{p, n-r}\left(T^{1 / 4} w ; f^{(r)}\right) .
$$

Corollary $15\left(\left[A_{5}\right]\right)$. Let $w=\exp (-Q) \in \mathcal{F}_{\lambda}\left(C^{r+2}+\right), 0<\lambda<(r+2) /(r+1)$, where $1 \leqslant r$ is an integer, and let (1.2) be satisfied. Let $1 \leqslant p \leqslant \infty$. We suppose that an absolutely continuous function $f^{(r-1)}$ satisfies $w f^{(r)} \in L^{p}(\mathbb{R})$. Then there exists a constant $C \geqslant 1$ such that if

$$
\|w(f-P)\|_{L^{p}(\mathbb{R})} \leqslant \varepsilon
$$


holds for some $n>r, P \in \mathcal{P}_{2 n}$ and $\varepsilon>0$, then

$$
\left\|\frac{w}{T^{(2 i+1) / 4}}\left(f^{(i)}-P^{(i)}\right)\right\|_{L^{p}(\mathbb{R})} \leqslant C\left\{E_{p, n-i}\left(w ; f^{(i)}\right)+\left(\frac{n}{a_{n}}\right)^{i} \varepsilon\right\}
$$

holds for all $i=1,2, \ldots, j$. In particular, if

$$
\|w(f-P)\|_{L^{p}(\mathbb{R})} \leqslant C E_{p, n}(w ; f)
$$

then

$$
\left\|\frac{w}{T^{(2 i+1) / 4}}\left(f^{(i)}-P^{(i)}\right)\right\|_{L^{p}(\mathbb{R})} \leqslant C\left(\frac{a_{n}}{n}\right)^{r-i} E_{p, n-r}\left(w ; f^{(r)}\right), \quad i=1,2, \ldots, r .
$$

Corollary $16\left(\left[A_{6}\right]\right)$. Let $w=\exp (-Q) \in \mathcal{F}_{\lambda}\left(C^{r+2}+\right), 0<\lambda<(r+2) /(r+1)$, where $1 \leqslant r$ is an integer, and let (1.2) be satisfied. Let $1 \leqslant p \leqslant \infty$. We suppose that an absolutely continuous function $f^{(r-1)}$ satisfies $T^{(2 r+1) / 4} w f^{(r)} \in L^{p}(\mathbb{R})$. Then there exists a constant $C \geqslant 1$ such that if

$$
\left\|T^{r / 2} w(f-P)\right\|_{L^{p}(\mathbb{R})} \leqslant \varepsilon
$$

holds for some $n>r, P \in \mathcal{P}_{2 n}$ and $\varepsilon>0$, then

$$
\left\|w\left(f^{(i)}-P^{(i)}\right)\right\|_{L^{p}(\mathbb{R})} \leqslant C\left\{E_{p, n-i}\left(T^{(2 i+1) / 4} w ; f^{(i)}\right)+\left(\frac{n}{a_{n}}\right)^{i} \varepsilon\right\}
$$

holds for all $i=1,2, \ldots, r$. In particular, if

$$
\left\|T^{r / 2} w(f-P)\right\|_{L^{p}(\mathbb{R})} \leqslant C E_{p, n}\left(T^{r / 2} w ; f\right),
$$

then

$$
\left\|w\left(f^{(i)}-P^{(i)}\right)\right\|_{L^{p}(\mathbb{R})} \leqslant C\left(\frac{a_{n}}{n}\right)^{r-i} E_{p, n-r}\left(T^{(2 r+1) / 4} w ; f^{(r)}\right) .
$$

Proof of Theorem 5. In Theorem 14 we set

$$
P=v_{n}, \quad \varepsilon=C E_{p, n}\left(T^{1 / 4} w, f\right),
$$

and then we have

$$
\left\|\frac{w}{T^{i / 2}}\left(f^{(i)}-v_{n}^{(i)}\right)\right\|_{L^{p}(\mathbb{R})} \leqslant C\left\{E_{p, n-i}\left(T^{1 / 4} w ; f^{(i)}\right)+C\left(\frac{n}{a_{n}}\right)^{i} E_{p, n}\left(T^{1 / 4} w, f\right)\right\} .
$$

From Results 4, 5, we obtain

$$
\begin{aligned}
& \left\|\frac{w}{T^{i / 2}}\left(f^{(i)}-v_{n}^{(i)}\right)\right\|_{L^{p}(\mathbb{R})} \leqslant C E_{p, n-i}\left(T^{1 / 4} w ; f^{(i)}\right)+C\left(\frac{a_{n}}{n}\right)^{r-i} E_{p, n-r}\left(T^{1 / 4} w ; f^{(r)}\right) \\
& \leqslant C\left(\frac{a_{n}}{n}\right)^{r-i} E_{p, n-r}\left(T^{1 / 4} w ; f^{(r)}\right) .
\end{aligned}
$$

Similarly, in Corollary 15 we set

$$
P=v_{n}, \quad \varepsilon=C E_{p, n}(w, f), \quad T^{-1 / 4} w \sim w_{-1 / 4},
$$

then we conclude

$$
\begin{aligned}
& \left\|\frac{w}{T^{(2 i+1) / 4}}\left(f^{(i)}-v_{n}^{(i)}\right)\right\|_{L^{p}(\mathbb{R})} \leqslant C\left\|\frac{w_{-1 / 4}}{T^{i / 2}}\left(f^{(i)}-v_{n}^{(i)}\right)\right\|_{L^{p}(\mathbb{R})} \\
& \leqslant C\left\{E_{p, n-i}\left(T^{1 / 4} w_{-1 / 4} ; f^{(i)}\right)+C\left(\frac{n}{a_{n}}\right)^{i} E_{p, n}\left(w_{-1 / 4}, f\right)\right\} \\
& \leqslant C\left\{E_{p, n-i}\left(w ; f^{(i)}\right)+C E_{p, n-i}\left(w, f^{(i)}\right)\right\} \\
& \leqslant C\left(\frac{a_{n}}{n}\right)^{r-i} E_{p, n-r}\left(w ; f^{(r)}\right) .
\end{aligned}
$$


Furthermore, in Corollary 16 we set

$$
P=v_{n}, \quad \varepsilon=C E_{p, n}\left(T^{r / 2} w, f\right), \quad T^{r / 2} w \sim w_{r / 2} .
$$

Then we have

$$
\left\|w\left(f^{(i)}-v_{n}^{(i)}\right)\right\|_{L^{p}(\mathbb{R})} \leqslant C\left(\frac{a_{n}}{n}\right)^{r-i} E_{p, n-r}\left(T^{(2 i+1) / 4} w ; f^{(r)}\right) .
$$

Consequently, we have the results.

To prove Theorem 6 we need Corollary 15 .

Proof of Theorem 6. In Corollary 15 we set

$$
P=v_{n}, \quad \varepsilon=C E_{p, n}(w, f),
$$

and then from Theorem 2 and Lemma 13, we have for $i=0, \ldots, r-1$

$$
\begin{aligned}
& \left\|\frac{w}{T^{(2 i+1) / 4}}\left(f^{(i)}-v_{n}^{(i)}\right)\right\|_{L^{p}(\mathbb{R})} \leqslant C E_{p, n-i}\left(w ; f^{(i)}\right) \leqslant C \tilde{\omega}_{r, p}\left(w, f^{(i)}, \frac{a_{n}}{n}\right) \\
& \leqslant C\left(\frac{a_{n}}{n}\right)^{r-i-1+\frac{1}{p}} O(1)^{1-1 / p}\left\{\int_{\mathbb{R}} w(y)\left|\mathrm{d} f^{(r-1)}\right|\right\}^{1 / p} .
\end{aligned}
$$

\section{Estimates for the Partial Sum of Fourier-type Expansion}

We prepare two lemmas.

Lemma 17 ([6]). The following is well-known.

$$
\left\|w\left(f-s_{n}(f)\right)\right\|_{L_{2}(\mathbb{R})}=E_{2, n}(f, w) .
$$

The following so-called Nikolskii-type inequality is useful.

Theorem $18\left(\left[A_{7}\right]\right)$. Let $w=\exp (-Q) \in \mathcal{F}_{\lambda}\left(C^{3}+\right)(0<\lambda<3 / 2)$, and let $P \in \mathcal{P}_{n}$. For $0<p \leqslant q \leqslant \infty$, we have

$$
\|w P\|_{L_{p}(\mathbb{R})} \leqslant C a_{n}^{\frac{1}{p}-\frac{1}{q}}\|w P\|_{L_{q}(\mathbb{R})}
$$

and for $1 \leqslant q<p \leqslant \infty$, we have

$$
\left\|T^{\frac{1}{2}\left(\frac{1}{p}-\frac{1}{q}\right)} w P\right\|_{L_{p}(\mathbb{R})} \leqslant C\left(\frac{n}{a_{n}}\right)^{\frac{1}{q}-\frac{1}{p}}\|w P\|_{L_{q}(\mathbb{R})},
$$

where $T$ is defined by (1.1).

Proof of Theorem \%. We see by Result 1 and Result 5,

$$
\begin{aligned}
& \left\|\frac{w}{T^{1 / 4}}\left(f-s_{n}\right)\right\|_{L^{p}(\mathbb{R})} \leqslant\left\|\frac{w}{T^{1 / 4}}\left(f-v_{n}\right)\right\|_{L^{p}(\mathbb{R})}+\left\|\frac{w}{T^{1 / 4}}\left(v_{n}-s_{n}\right)\right\|_{L^{p}(\mathbb{R})} \\
& \leqslant C E_{p, n}(w, f)+\left\|\frac{w}{T^{1 / 4}}\left(v_{n}-s_{n}\right)\right\|_{L^{p}(\mathbb{R})} \\
& \leqslant C\left(\frac{a_{n}}{n}\right)\left\|w f^{\prime}\right\|_{L^{p}(\mathbb{R})}+\left\|\frac{w}{T^{1 / 4}}\left(v_{n}-s_{n}\right)\right\|_{L^{p}(\mathbb{R})} .
\end{aligned}
$$

First, let $1 \leqslant p<2$. By Theorem 18 (5.1) with $q=2$,

$$
\begin{aligned}
& \left\|\frac{w}{T^{1 / 4}}\left(v_{n}-s_{n}\right)\right\|_{L_{p}(\mathbb{R})} \leqslant\left\|w\left(v_{n}-s_{n}\right)\right\|_{L_{p}(\mathbb{R})} \\
& \leqslant C a_{n}^{\frac{1}{p}-\frac{1}{2}}\left\|w\left(v_{n}-s_{n}\right)\right\|_{L_{2}(\mathbb{R})} .
\end{aligned}
$$


We estimate

$$
\left\|w\left(v_{n}-s_{n}\right)\right\|_{L_{2}(\mathbb{R})} \leqslant\left\|w\left(f-v_{n}\right)\right\|_{L_{2}(\mathbb{R})}+\left\|w\left(f-s_{n}\right)\right\|_{L_{2}(\mathbb{R})} .
$$

From Lemma 17 we see

$$
\left\|w\left(f-s_{n}\right)\right\|_{L_{2}(\mathbb{R})}=E_{2, n}(f, w) .
$$

Hence, by Result 5 we see that

$$
\left\|w\left(f-s_{n}\right)\right\|_{L_{2}(\mathbb{R})} \leqslant C\left(\frac{a_{n}}{n}\right)\left\|w f^{\prime}\right\|_{L^{2}(\mathbb{R})} .
$$

This estimate also implies

$$
\begin{aligned}
& \left\|w\left(f-v_{n}\right)\right\|_{L_{2}(\mathbb{R})} \leqslant\left\|\left(\frac{1}{n} \sum_{j=n+1}^{2 n} f-\frac{1}{n} \sum_{j=n+1}^{2 n} s_{j}(f)\right) w\right\|_{L^{2}(\mathbb{R})} \\
& \leqslant \frac{1}{n} \sum_{j=n+1}^{2 n}\left\|w\left(f-s_{j}(f)\right)\right\|_{L^{2}(\mathbb{R})} \\
& \leqslant \frac{1}{n} \sum_{j=n+1}^{2 n} C\left(\frac{a_{j}}{j}\right)\left\|w f^{\prime}\right\|_{L^{2}(\mathbb{R})} \\
& \leqslant C\left(\frac{a_{n}}{n}\right)\left\|w f^{\prime}\right\|_{L^{2}(\mathbb{R})},
\end{aligned}
$$

so that we see

$$
\left\|w\left(v_{n}-s_{n}\right)\right\|_{L_{2}(\mathbb{R})} \leqslant C\left(\frac{a_{n}}{n}\right)\left\|w f^{\prime}\right\|_{L^{2}(\mathbb{R})} .
$$

Substituting it into (5.4), we obtain

$$
\left\|\frac{w}{T^{1 / 4}}\left(v_{n}-s_{n}\right)\right\|_{L_{p}(\mathbb{R})} \leqslant C a_{n}^{\frac{1}{p}-\frac{1}{2}}\left(\frac{a_{n}}{n}\right)\left\|w f^{\prime}\right\|_{L^{2}(\mathbb{R})} .
$$

Therefore, by (5.3)

$$
\begin{aligned}
& \left\|\frac{w}{T^{1 / 4}}\left(f-s_{n}\right)\right\|_{L^{p}(\mathbb{R})} \leqslant C\left(\frac{a_{n}}{n}\right)\left\|w f^{\prime}\right\|_{L^{p}(\mathbb{R})}+C a_{n}^{\frac{1}{p}-\frac{1}{2}}\left(\frac{a_{n}}{n}\right)\left\|w f^{\prime}\right\|_{L^{2}(\mathbb{R})} \\
& \leqslant C a_{n}^{\frac{1}{p}-\frac{1}{2}}\left(\frac{a_{n}}{n}\right)\left\|w f^{\prime}\right\|_{L^{2}(\mathbb{R})} .
\end{aligned}
$$

Next, in Theorem 18 (5.2) we set $q=2$ and $2 \leqslant p \leqslant \infty$. Then we have

$$
\begin{aligned}
& \left\|\frac{w}{T^{1 / 4}}\left(v_{n}(f)-s_{n}(f)\right)\right\|_{L_{p}(\mathbb{R})} \leqslant\left\|T^{\frac{1}{2}\left(\frac{1}{p}-\frac{1}{2}\right)} w\left(v_{n}(f)-s_{n}(f)\right)\right\|_{L_{p}(\mathbb{R})} \\
& \leqslant C\left(\frac{n}{a_{n}}\right)^{\frac{1}{2}-\frac{1}{p}}\left\|w\left(v_{n}(f)-s_{n}(f)\right)\right\|_{L_{2}(\mathbb{R}) .}
\end{aligned}
$$

Hence, by (5.6) we have

$$
\left\|\frac{w}{T^{1 / 4}}\left(v_{n}(f)-s_{n}(f)\right)\right\|_{L_{p}(\mathbb{R})} \leqslant C\left(\frac{n}{a_{n}}\right)^{\frac{1}{2}-\frac{1}{p}}\left(\frac{a_{n}}{n}\right)\left\|w f^{\prime}\right\|_{L^{2}(\mathbb{R})} .
$$

Consequently, by (5.3) we have

$$
\left\|\frac{w}{T^{1 / 4}}\left(f-s_{n}\right)\right\|_{L^{p}(\mathbb{R})} \leqslant C\left(\frac{a_{n}}{n}\right)^{\frac{1}{2}+\frac{1}{p}}\left\|w f^{\prime}\right\|_{L^{2}(\mathbb{R})} .
$$

Therefore, by (5.7) and (5.8) we see

$$
\begin{aligned}
& \left\|\frac{w}{T^{1 / 4}}\left(f-s_{n}\right)\right\|_{L^{p}(\mathbb{R})} \leqslant C\left\{a_{n}^{\frac{1}{p}-\frac{1}{2}}\left(\frac{a_{n}}{n}\right)\left\|w f^{\prime}\right\|_{L^{2}(\mathbb{R})}+\left(\frac{a_{n}}{n}\right)^{\frac{1}{2}+\frac{1}{p}}\left\|w f^{\prime}\right\|_{L^{2}(\mathbb{R})}\right\} \\
& \leqslant C\left(\frac{a_{n}}{n}\right)^{\frac{1}{2}+\frac{1}{p}}\left\|w f^{\prime}\right\|_{L^{2}(\mathbb{R})} \begin{cases}n^{\frac{1}{p}-\frac{1}{2}}, & 1 \leqslant p<2 ; \\
1, & 2 \leqslant p \leqslant \infty,\end{cases}
\end{aligned}
$$

that is, we have the result. 
Proposition 19 ([10, Lemma 2.5]). Let $1 \leqslant p \leqslant \infty$ and $w \in \mathcal{F}_{\lambda}\left(C^{3}+\right)(0<\lambda<3 / 2)$. Then there exists a constant $C>0$ such that if $P \in \mathcal{P}_{n}(n \in \mathbb{N})$, then we have

$$
\left\|\frac{w}{T^{j / 2}} P^{(j)}\right\|_{L^{p}(\mathbb{R})} \leqslant C\left(\frac{n}{a_{n}}\right)^{j}\|w P\|_{L^{p}(\mathbb{R})}, j \in \mathbb{N} .
$$

And also if we assume that $w \in \mathcal{F}_{\lambda}\left(C^{4}+\right)(0<\lambda<4 / 3)$, then there exists a constant $C_{2}>0$ such that

$$
\left\|w P^{(j)}\right\|_{L^{p}(\mathbb{R})} \leqslant C_{2}\left(\frac{n}{a_{n}}\right)^{j}\left\|T^{j / 2} w P\right\|_{L^{p}(\mathbb{R})}, j \in \mathbb{N}
$$

holds.

Proof of Theorem 8. Let $i=0,1, \ldots, r-1$. By Theorem 6 we see

$$
\begin{aligned}
& \left\|\frac{w}{T^{(2 i+1) / 4}}\left(f^{(i)}-s_{n}^{(i)}\right)\right\|_{L^{p}(\mathbb{R})} \leqslant\left\|\frac{w}{T^{(2 i+1) / 4}}\left(f^{(i)}-v_{n}^{(i)}\right)\right\|_{L^{p}(\mathbb{R})}+\left\|\frac{w}{T^{(2 i+1) / 4}}\left(v_{n}^{(i)}-s_{n}^{(i)}\right)\right\|_{L^{p}(\mathbb{R})} \\
& \leqslant C\left(\frac{a_{n}}{n}\right)^{r-i-1+\frac{1}{p}} O(1)^{1-1 / p}\left\{\int_{\mathbb{R}} w(y)\left|\mathrm{d} f^{(r-1)}\right|\right\}^{1 / p}+\left\|\frac{w}{T^{(2 i+1) / 4}}\left(v_{n}^{(i)}-s_{n}^{(i)}\right)\right\|_{L^{p}(\mathbb{R})} .
\end{aligned}
$$

We estimate the second term in (5.9). From Proposition 19 and Result 4 we have

$$
\left\|\frac{w}{T^{(2 i+1) / 4}}\left(v_{n}^{(i)}-s_{n}^{(i)}\right)\right\|_{L_{p}(\mathbb{R})} \leqslant\left(\frac{n}{a_{n}}\right)^{i}\left\|\frac{w}{T^{1 / 4}}\left(v_{n}-s_{n}\right)\right\|_{L_{p}(\mathbb{R})}
$$

First, let $1 \leqslant p<2$. By Theorem 18 (5.1) with $q=2$,

$$
\left\|\frac{w}{T^{1 / 4}}\left(v_{n}-s_{n}\right)\right\|_{L_{p}(\mathbb{R})} \leqslant\left\|w\left(v_{n}-s_{n}\right)\right\|_{L_{p}(\mathbb{R})} \leqslant C a_{n}^{\frac{1}{p}-\frac{1}{2}}\left\|w\left(v_{n}-s_{n}\right)\right\|_{L_{2}(\mathbb{R})} .
$$

We estimate

$$
\left\|w\left(v_{n}-s_{n}\right)\right\|_{L_{2}(\mathbb{R})} \leqslant\left\|w\left(f-v_{n}\right)\right\|_{L_{2}(\mathbb{R})}+\left\|w\left(f-s_{n}\right)\right\|_{L_{2}(\mathbb{R})} .
$$

From Lemma 17,

$$
\left\|w\left(f-s_{n}\right)\right\|_{L_{2}(\mathbb{R})}=E_{2, n}(f, w) \leqslant C \tilde{\omega}_{r, 2}\left(f, w, L \frac{a_{n}}{n}\right) .
$$

Hence, by Theorem 2 with $p=2$ we see

$$
\left\|w\left(f-s_{n}\right)\right\|_{L_{2}(\mathbb{R})} \leqslant C\left(\frac{a_{n}}{n}\right)^{r-\frac{1}{2}} O(1)^{\frac{1}{2}}\left(\int_{\mathbb{R}} w(y)\left|\mathrm{d} f^{(r-1)}(y)\right|\right)^{1 / 2} .
$$

This estimate also implies

$$
\begin{aligned}
& \left\|w\left(f-v_{n}\right)\right\|_{L_{2}(\mathbb{R})} \leqslant\left\|\left(\frac{1}{n} \sum_{j=n+1}^{2 n} f-\frac{1}{n} \sum_{j=n+1}^{2 n} s_{j}(f)\right) w\right\|_{L^{2}(\mathbb{R})} \\
& \leqslant \frac{1}{n} \sum_{j=n+1}^{2 n}\left\|w\left(f-s_{j}(f)\right)\right\|_{L^{2}(\mathbb{R})} \\
& \leqslant \frac{1}{n} \sum_{j=n+1}^{2 n} C\left(\frac{a_{n}}{n}\right)^{r-\frac{1}{2}} O(1)^{\frac{1}{2}}\left(\int_{\mathbb{R}} w(y)\left|\mathrm{d} f^{(r-1)}(y)\right|\right)^{1 / 2} \\
& \leqslant C\left(\frac{a_{n}}{n}\right)^{r-\frac{1}{2}} O(1)^{\frac{1}{2}}\left(\int_{\mathbb{R}} w(y)\left|\mathrm{d} f^{(r-1)}(y)\right|\right)^{1 / 2},
\end{aligned}
$$

so that we see

$$
\left\|w\left(v_{n}-s_{n}\right)\right\|_{L_{2}(\mathbb{R})} \leqslant C\left(\frac{a_{n}}{n}\right)^{r-\frac{1}{2}} o(1)^{\frac{1}{2}}\left(\int_{\mathbb{R}} w(y)\left|\mathrm{d} f^{(r-1)}(y)\right|\right)^{1 / 2} .
$$


Substituting it into (5.11), we obtain

$$
\left\|\frac{w}{T^{1 / 4}}\left(v_{n}-s_{n}\right)\right\|_{L_{p}(\mathbb{R})} \leqslant C a_{n}^{\frac{1}{p}-\frac{1}{2}}\left(\frac{a_{n}}{n}\right)^{r-\frac{1}{2}} o(1)^{\frac{1}{2}}\left(\int_{\mathbb{R}} w(y)\left|\mathrm{d} f^{(r-1)}(y)\right|\right)^{1 / 2} .
$$

Next, let $2 \leqslant p \leqslant \infty$, and in Theorem 18 (5.2) we set $q=2$. Then we have

$$
\begin{aligned}
& \left\|\frac{w}{T^{1 / 4}}\left(v_{n}(f)-s_{n}(f)\right)\right\|_{L_{p}(\mathbb{R})} \leqslant\left\|T^{\frac{1}{2}\left(\frac{1}{p}-\frac{1}{2}\right)} w\left(v_{n}(f)-s_{n}(f)\right)\right\|_{L_{p}(\mathbb{R})} \\
& \leqslant C\left(\frac{n}{a_{n}}\right)^{\frac{1}{2}-\frac{1}{p}}\left\|w\left(v_{n}(f)-s_{n}(f)\right)\right\|_{L_{2}(\mathbb{R})} .
\end{aligned}
$$

Hence, by (5.12) we have

$$
\begin{aligned}
& \left\|\frac{w}{T^{1 / 4}}\left(v_{n}(f)-s_{n}(f)\right)\right\|_{L_{p}(\mathbb{R})} \leqslant C\left(\frac{n}{a_{n}}\right)^{\frac{1}{2}-\frac{1}{p}}\left(\frac{a_{n}}{n}\right)^{r-\frac{1}{2}} o(1)^{\frac{1}{2}}\left(\int_{\mathbb{R}} w(y)\left|\mathrm{d} f^{(r-1)}(y)\right|\right)^{1 / 2} \\
& \leqslant C\left(\frac{a_{n}}{n}\right)^{r-1+\frac{1}{p}} O(1)^{\frac{1}{2}}\left(\int_{\mathbb{R}} w(y)\left|\mathrm{d} f^{(r-1)}(y)\right|\right)^{1 / 2} .
\end{aligned}
$$

Therefore, (5.13) and (5.14) mean

$$
\begin{aligned}
& \left\|\frac{w}{T^{1 / 4}}\left(v_{n}(f)-s_{n}(f)\right)\right\|_{L_{p}(\mathbb{R})} \\
& \leqslant C\left(\frac{a_{n}}{n}\right)^{r-1+\frac{1}{p}} O(1)^{\frac{1}{2}}\left(\int_{\mathbb{R}} w(y)\left|\mathrm{d} f^{(r-1)}(y)\right|\right)^{1 / 2} \begin{cases}n^{\frac{1}{p}-\frac{1}{2}}, & 1 \leqslant p<2 \\
1, & 2 \leqslant p \leqslant \infty\end{cases}
\end{aligned}
$$

Hence, from (5.10) we see

$$
\begin{aligned}
& \left\|\frac{w}{T^{(2 i+1) / 4}}\left(v_{n}^{(i)}-s_{n}^{(i)}\right)\right\|_{L_{p}(\mathbb{R})} \\
& \leqslant C\left(\frac{a_{n}}{n}\right)^{r-i-1+\frac{1}{p}} o(1)^{\frac{1}{2}}\left(\int_{\mathbb{R}} w(y)\left|\mathrm{d} f^{(r-1)}(y)\right|\right)^{1 / 2} \begin{cases}n^{\frac{1}{p}-\frac{1}{2}}, & 1 \leqslant p<2 ; \\
1, & 2 \leqslant p \leqslant \infty .\end{cases}
\end{aligned}
$$

Substituting it for (5.9),

$$
\begin{aligned}
& \left\|\frac{w}{T^{(2 i+1) / 4}}\left(f^{(i)}-s_{n}^{(i)}\right)\right\|_{L^{p}(\mathbb{R})} \\
& \leqslant C\left(\frac{a_{n}}{n}\right)^{r-i-1+\frac{1}{p}} O(1)^{1-1 / p}\left\{\int_{\mathbb{R}} w(y)\left|\mathrm{d} f^{(r-1)}\right|\right\}^{1 / p} \\
& \quad+C\left(\frac{a_{n}}{n}\right)^{r-i-1+\frac{1}{p}} O(1)^{\frac{1}{2}}\left(\int_{\mathbb{R}} w(y)\left|\mathrm{d} f^{(r-1)}(y)\right|\right)^{1 / 2} \begin{cases}n^{\frac{1}{p}-\frac{1}{2}}, & 1 \leqslant p<2 ; \\
1, & 2 \leqslant p \leqslant \infty\end{cases} \\
& \leqslant C\left(\frac{a_{n}}{n}\right)^{r-i-1+\frac{1}{p}}\left\{\int_{\mathbb{R}} w(y)\left|\mathrm{d} f^{(r-1)}\right|\right\}^{1 / p} \begin{cases}o(1)^{1-1 / p} n^{\frac{1}{p}-\frac{1}{2}}, & 1 \leqslant p<2 ; \\
o(1)^{\frac{1}{2}}, & 2 \leqslant p \leqslant \infty .\end{cases}
\end{aligned}
$$

Consequently, the proof of Theorem 8 is complete.

\section{References}

1. S. B. Damelin and D. S. Lubinsky, Jackson Theorems for Erdös Weights in $L_{p}(0<p \leqslant \infty)$, Journal of Approximation Theory 94(1998), 333-382.

2. H. S. Jung and R. Sakai, Higher order derivatives of approximation polynomial on $\mathbb{R}$, Journal of inequalities and applications, 2015: 268, DOI 10.1186/s13660-o15-0789-y.

3. D. S. Lubinsky, A Survey of Weighted Polynomial Approximation with Exponential Weights, Surveys on Approximation Theory, 3(2007), 1-105.

4. A. L. Levin and D. S. Lubinsky, Orthogonal Polynomials for Exponential Weights, Springer, New York, 2001.

5. H. N. Mhaskar, Extensions of the Dirichlet-Jordan Convergence Criterion to a General Class of Orthogonal Polynomial Expansions, Journal of Approximation Theory 42 (1984), 138-148. 
6. W. Rudin, Principles of Mathematical Analysis, McGraw-Hill, New York, 1964.

7. R. Sakai and N. Suzuki, Favard-type inequalities for exponential weights, Pioneer J. of Math. vol 3. No.1, 2011, 1-16.

8. R. Sakai and N. Suzuki, Mollification of exponential weights and its application to the Markov-Bernstein inequality, Pioneer J. of Math., Vol.7, no.1, pp.83-101, 2013.

9. K. Itoh, R. Sakai and N. Suzuki, The de la Vallée Poussin Mean and Polynomial Appoximation for Exponential Weight, International Journal of Analysis, 2015, Article ID 706930, 8 pages, (2015).

10. K. Itoh, R. Sakai and N. Suzuki, An estimate for derivative of the de la Vallée Poussin mean, Math. J. Ibaraki Univ. 47 (2015), 1-18.

11. K. Itoh, R. Sakai and N. Suzuki, Uniform convergence of orthogonal polynomial expansions for exponential weights, preprint.

12. K. Itoh, R. Sakai and N. Suzuki, Polynomial approximation for absolutely continuous functions, accepted by Tohoku Math. Journal.

\section{A Appendix}

In this appendix, we give proofs of lemmas (propositions, theorems) which are stated in previous sections.

\section{Proof of Theorem 9}

In this section we will prove Theorem 9. We use the method of [5, Lemma 3.4.4]. First, we need a lemma. In $[8$, Theorem 4.2], if we put $\mu=\nu=\alpha=0, \beta \in \mathbb{R}$, then we obtain the following proposition.

Proposition 20 (cf. [8, Theorem 4.2]). Let $r$ be a positive integer, $0<\lambda<(r+2) /(r+1)$ and let $w=$ $\exp (-Q) \in \mathcal{F}_{\lambda}\left(C^{r+2}+\right)$. Then for $\beta \in \mathbb{R}$, we can construct a new weight $w_{\beta} \in \mathcal{F}_{\lambda}\left(C^{r+1}+\right)\left(\subset \mathcal{F}_{\lambda}\left(C^{2}+\right)\right)$ such that $\left(1+\left|Q^{\prime}(x)\right|\right)^{\beta} w(x) \sim w_{\beta}$ on $\mathbb{R}, a_{n}\left(w_{\beta}\right) \sim a_{n}(w)$ on $\mathbb{N}$ and $T_{w_{\beta}}(x) \sim T_{w}(x)$ on $\mathbb{R}$ hold.

$\left[A_{1}\right]$ (Proof of Theorem 9). Since

$$
\lim _{t \rightarrow \infty} \sup \frac{Q^{\prime \prime}(t)}{Q^{\prime}(t)^{2}} \leqslant C \lim _{t \rightarrow \infty} \frac{Q^{\prime}(t)}{Q(t) Q^{\prime}(t)^{2}} \leqslant C \lim _{t \rightarrow \infty} \frac{1}{Q(t) Q^{\prime}(t)}=0 .
$$

Therefore,

$$
\left(Q^{\prime} w\right)^{\prime}(t)=\left(Q^{\prime \prime}(t)-Q^{\prime}(t)^{2}\right) w(t)<0
$$

for large values of $t$, and thus, $Q^{\prime} w$ is decreasing for $t$ large enough, that is, $Q^{\prime} w$ is bounded on $\mathbb{R}$. So, we may show (3.1) only for large positive values of $x$. By (A.1) we may find a constant $A$, whose value will be retained during the remainder of this proof, such that $Q^{\prime \prime}(t) \leqslant(1 / 2) Q^{\prime}(t)^{2}$ if $t \geqslant A$. Then an integration by parts shows that for $x \geqslant A$,

$$
\begin{aligned}
& \int_{A}^{x} w^{-1}(t) \mathrm{d} t=\int_{A}^{x} Q^{\prime}(t)^{-1} Q^{\prime}(t) e^{Q(t)} \mathrm{d} t \\
& \leqslant Q^{\prime}(x)^{-1} e^{Q(x)}+\int_{A}^{x} \frac{Q^{\prime \prime}(t)}{Q^{\prime}(t)^{2}} w^{-1}(t) \mathrm{d} t \leqslant Q^{\prime}(x)^{-1} e^{Q(x)}+\frac{1}{2} \int_{A}^{x} w^{-1}(t) \mathrm{d} t .
\end{aligned}
$$

Therefore,

$$
\int_{A}^{x} w^{-1}(t) \mathrm{d} t \leqslant 2 Q^{\prime}(x) w^{-1}(x), \quad x \geqslant A,
$$

that is,

$$
Q^{\prime}(x) w(x) \int_{A}^{x} w^{-1}(t) \mathrm{d} t \leqslant 2, \quad x \geqslant A .
$$

Since $Q^{\prime}(x) w(x)$ is bounded on $\mathbb{R}$,

$$
Q^{\prime}(x) w(x) \int_{0}^{A} w^{-1}(t) \mathrm{d} t \leqslant C, \quad x \geqslant A .
$$


Hence, we complete the proof of (3.1). To prove the second part (b) we may put $\gamma=0$. For almost all $x \geqslant 0$,

$$
\begin{aligned}
& \left|Q^{\prime}(x) w(x) g(x)\right|=\left|Q^{\prime}(x) w(x) \int_{0}^{x} w^{-1}(t) w(t) g^{\prime}(t) \mathrm{d} t\right| \\
& \leqslant\left\|w g^{\prime}\right\|_{L^{\infty}(x \geqslant 0)}\left|Q^{\prime}(x) w(x) \int_{0}^{x} w^{-1}(t) \mathrm{d} t\right| \leqslant C\left\|w g^{\prime}\right\|_{L^{\infty}(x \geqslant 0)}
\end{aligned}
$$

by (3.1). This completes the proof of (3.2) in the case of $p=\infty$. We observe that

$$
\begin{aligned}
& \int_{0}^{\infty} Q^{\prime}(x) w(x)|g(x)| \mathrm{d} x \leqslant \int_{0}^{\infty} Q^{\prime}(x) w(x) \int_{0}^{x}\left|g^{\prime}(t)\right| \mathrm{d} t \mathrm{~d} x \\
& =\int_{0}^{\infty}\left|g^{\prime}(t)\right| \int_{t}^{\infty} Q^{\prime}(x) w(x) \mathrm{d} x \mathrm{~d} t=\int_{0}^{\infty} w(t)\left|g^{\prime}(t)\right| \mathrm{d} t .
\end{aligned}
$$

Similarly,

$$
\int_{-\infty}^{0}\left|Q^{\prime}(x) w(x) g(x)\right| \mathrm{d} x \leqslant \int_{-\infty}^{0} w(t)\left|g^{\prime}(t)\right| \mathrm{d} t .
$$

This proves (3.2) in the case of $p=1$. We complete the proof of other values of $p$ by applying the Riesz-Thorin interpolation theorem to the operator on $x \geqslant 0$

$$
\phi \rightarrow Q^{\prime}(x) w(x) \int_{0}^{x} w^{-1}(t) \phi(t) \mathrm{d} t
$$

and similarly, to the operator on $(-\infty, 0]$

$$
\phi \rightarrow Q^{\prime}(x) w(x) \int_{x}^{0} w^{-1}(t) \phi(t) \mathrm{d} t .
$$

We will show (3.3). We apply Result 7 to

$$
\mu=\nu=\alpha=0, \quad \beta=1,2, \ldots, r-1 .
$$

Let $w(x)=\exp (-Q(x)) \in \mathcal{F}\left(C^{2}+\right)$. Then there exist

$$
\begin{aligned}
& Q^{\prime}(x) w(x) \sim w_{1}(x)=\exp \left(-Q_{1}(x)\right), \\
& Q_{1}^{\prime}(x) w_{1}(x) \sim w_{2}(x)=\exp \left(-Q_{2}(x)\right), \\
& \quad \cdots \ldots \ldots \ldots \ldots . . . \\
& Q_{r-2}^{\prime}(x) w_{r-2}(x) \sim w_{r-1}(x)=\exp \left(-Q_{r-1}(x)\right),
\end{aligned}
$$

and

$$
Q_{i}^{(j)}(x) \sim Q^{(j)}(x), \quad i=1,2, \ldots, r-1, \quad j=0,1 .
$$

Hence we see

$$
\begin{aligned}
& \left\|w g^{(r)}\right\|_{L^{p}(\mathbb{R})} \geqslant C_{1}\left\|Q^{\prime} w g^{(r-1)}\right\|_{L^{p}(\mathbb{R})} \sim\left\|w_{1} g^{(r-1)}\right\|_{L^{p}(\mathbb{R})} \\
& \geqslant C_{2}\left\|Q_{1}^{\prime} w_{1} g^{(r-2)}\right\|_{L^{p}(\mathbb{R})} \sim\left\|w_{2} g^{(r-2)}\right\|_{L^{p}(\mathbb{R})} \\
& \ldots \ldots \ldots \ldots \ldots \ldots \ldots \ldots \ldots \ldots \ldots \ldots \ldots \ldots \ldots \ldots \\
& \geqslant C_{r}\left\|Q_{r-1}^{\prime} w_{r-1} g\right\|_{L^{p}(\mathbb{R})} .
\end{aligned}
$$

Here we know

$$
\begin{aligned}
& Q_{i}^{\prime}(x) \sim Q^{\prime}(x), \quad i=1,2, \ldots, r-1 \\
& w_{r-1}(x) \sim Q_{r-2}^{\prime}(x) w_{r-2}(x) \sim \ldots \ldots \sim Q_{r-2}^{\prime}(x) Q_{r-3}^{\prime}(x) \ldots Q^{\prime}(x) w(x),
\end{aligned}
$$


that is,

$$
\left|Q_{r-1}^{\prime}(x) w_{r-1}(x)\right| \sim\left|Q^{\prime}(x)\right|^{r} w(x) .
$$

Consequently, we have (3.3), that is,

$$
\left\|\left(Q^{\prime}\right)^{r} w g\right\|_{L^{p}(|x| \geqslant \gamma)} \leqslant\left\|Q^{\prime r} w g\right\|_{L^{p}(\mathbb{R})} \leqslant C\left\|w g^{(r)}\right\|_{L^{p}(\mathbb{R})} .
$$

\section{Proofs of Theorems 11 and 12}

$\left[A_{2}\right]$ (Proof of Theorem 11). We see

$$
\begin{aligned}
& \left\|w \Delta_{h \Phi_{t}(x)}^{r}(f, x)\right\|_{L^{p}(|x| \leqslant \sigma(2 t))}^{p} \\
& \leqslant\left\|w \Delta_{h \Phi_{t}(x)}^{r}(f, x)\right\|_{L^{\infty}(|x| \leqslant \sigma(2 t))}^{p-1}\left\|w \Delta_{h \Phi_{t}(x)}^{r}(f, x)\right\|_{L^{1}(|x| \leqslant \sigma(2 t))} \\
& \leqslant\left[\left\|w \Delta_{h \Phi_{t}(x)}^{r}(f, x)\right\|_{L^{\infty}(|x| \leqslant \sigma(2 t))}+\left\|w\left(f-P_{r-1, f}\right)\right\|_{L^{\infty}(|x| \geqslant \sigma(4 t))}\right]^{p-1} \\
& \quad \times\left[\left\|w \Delta_{h \Phi_{t}(x)}^{r}(f, x)\right\|_{L^{1}(|x| \leqslant \sigma(2 t))}+\left\|w\left(f-P_{r-1, f}\right)\right\|_{L^{1}(|x| \geqslant \sigma(4 t))}\right] \\
& \leqslant \tilde{\omega}_{r, \infty}^{p-1}(f, w, t) \tilde{\omega}_{r, 1}(f, w, t) .
\end{aligned}
$$

On the other hand, since

$$
\left.\left\|w\left(f-P_{r-1, f}\right)\right\|_{L^{p}(|x| \geqslant \sigma(4 t))}^{p} \leqslant\left\|w\left(f-P_{r-1, f}\right)\right\|_{L^{\infty}(|x| \geqslant \sigma(4 t))}\right]^{p-1}\left[\left\|w\left(f-P_{r-1, f}\right)\right\|_{\left.L^{1}(|x| \geqslant \sigma(4 t))\right]}\right]
$$

we have

$$
\begin{aligned}
& \left\|w\left(f-P_{r-1, f}\right)\right\|_{L^{p}(|x| \geqslant \sigma(4 t))}^{p} \\
& \leqslant\left[\left\|w \Delta_{h \Phi_{t}(x)}^{r}(f, x)\right\|_{L^{\infty}(|x| \leqslant \sigma(2 t))}+\left\|w\left(f-P_{r-1, f}\right)\right\|_{L^{\infty}(|x| \geqslant \sigma(4 t))}\right]^{p-1} \\
& \quad \times\left[\left\|w \Delta_{h \Phi_{t}(x)}^{r}(f, x)\right\|_{L^{1}(|x| \leqslant \sigma(2 t))}+\left\|w\left(f-P_{r-1, f}\right)\right\|_{L^{1}(|x| \geqslant \sigma(4 t))}\right] \\
& \leqslant \tilde{\omega}_{r, \infty}^{p-1}(f, w, t) \tilde{\omega}_{r, 1}(f, w, t) .
\end{aligned}
$$

Hence,

$$
\tilde{\omega}_{r, p}(f, w, t) \leqslant \tilde{\omega}_{r, \infty}^{1-1 / p}(f, w, t) \tilde{\omega}_{r, 1}^{1 / p}(f, w, t)
$$

To prove Theorem 12 we use Theorem 9.

$\left[A_{3}\right]$ (Proof of Theorem 12). Let $w \in \mathcal{F}_{\lambda}\left(C^{3}+\right), \quad(0<\lambda<3 / 2)$. And $f^{(r-1)}(x)$ is continuous and has a bounded variation on any compact interval of $\mathbb{R}$. Furthermore, we suppose (1.8). We set

$$
g_{r-1}(x):=f^{(r-1)}\left(x+h \Phi_{t}(x) / 2\right)-f^{(r-1)}\left(x-h \Phi_{t}(x) / 2\right) .
$$

We use Hölder's inequality. Let $\frac{1}{p}+\frac{1}{q}=1, p, q \geqslant 1$. We see

$$
\begin{aligned}
& \left\|w \Delta_{h \Phi_{t}(x)}^{r}(f, x)\right\|_{L^{1}(|x| \leqslant \sigma(2 t))} \\
& =\int_{|x| \leqslant \sigma(2 t)}\left|w(x) \int_{-h \Phi_{t}(x) / 2}^{h \Phi_{t}(x) / 2} \ldots \int_{-h \Phi_{t}(x) / 2}^{h \Phi_{t}(x) / 2} g_{r-1}\left(x+t_{1}+\ldots+t_{r-1}\right) \mathrm{d} t_{1} \ldots \mathrm{d} t_{r-1}\right| \mathrm{d} x \\
& \leqslant C \int_{|x| \leqslant \sigma(2 t)} \int_{-h \Phi_{t}(x) / 2}^{h \Phi_{t}(x) / 2} \ldots \int_{-h \Phi_{t}(x) / 2}^{h \Phi_{t}(x) / 2} w\left(x+t_{1}+\ldots+t_{r-1}\right) \\
& \quad \times\left|g_{r-1}\left(x+t_{1}+\ldots+t_{r-1}\right)\right| \mathrm{d} t_{1} \ldots \mathrm{d} t_{r-1} \mathrm{~d} x \\
& \leqslant C \int_{|x| \leqslant \sigma(2 t)} \int_{-h}^{h} \ldots \int_{-h}^{h} w\left(x+t_{1}+\ldots+t_{r-1}\right)\left|g_{r-1}\left(x+t_{1}+\ldots+t_{r-1}\right)\right| \mathrm{d} t_{1} \ldots \mathrm{d} t_{r-1} \mathrm{~d} x
\end{aligned}
$$




$$
\begin{aligned}
& \leqslant C \int_{|x| \leqslant \sigma(2 t)}\left\{\int_{-h}^{h} 1^{q} \mathrm{~d} t_{r-1}\right\}^{1 / q} \\
& \times\left\{\int _ { - h } ^ { h } \left[\int_{-h}^{h} \ldots \int_{-h}^{h} w\left(x+t_{1}+\ldots+t_{r-1}\right)\left|g_{r-1}\left(x+t_{1}+\ldots+t_{r-1}\right)\right|\right.\right. \\
& \left.\left.\times \mathrm{d} t_{1} \ldots \mathrm{d} t_{r-2}\right]^{p} \mathrm{~d} t_{r-1}\right\}^{1 / p} \mathrm{~d} x \\
& \leqslant C \int_{|x| \leqslant \sigma(2 t)}\left\{\int_{-h}^{h} 1^{q} \mathrm{~d} t_{r-1}\right\}^{1 / q}\left\{\int_{-h}^{h} 1^{q} \mathrm{~d} t_{r-2}\right\}^{1 / q} \\
& \times\left\{\int _ { - h } ^ { h } \int _ { - h } ^ { h } \left[\int_{-h}^{h} \ldots \int_{-h}^{h} w\left(x+t_{1}+\ldots+t_{r-1}\right)\left|g_{r-1}\left(x+t_{1}+\ldots+t_{r-1}\right)\right|\right.\right. \\
& \left.\left.\times \mathrm{d} t_{1} \ldots \mathrm{d} t_{r-3}\right]^{p} \mathrm{~d} t_{r-1} \mathrm{~d} t_{r-2}\right\}^{1 / p} \mathrm{~d} x \\
& \leqslant C \int_{|x| \leqslant \sigma(2 t)}\left\{\int_{-h}^{h} 1^{q} \mathrm{~d} t_{r-1}\right\}^{1 / q}\left\{\int_{-h}^{h} 1^{q} \mathrm{~d} t_{r-2}\right\}^{1 / q} \ldots .\left\{\int_{-h}^{h} 1^{q} \mathrm{~d} t_{1}\right\}^{1 / q} \\
& \times\left\{\int_{-h}^{h} \int_{-h}^{h} \ldots \int_{-h}^{h}\left|w\left(x+t_{1}+\ldots+t_{r-1}\right) g_{r-1}\left(x+t_{1}+\ldots+t_{r-1}\right)\right|^{p}\right. \\
& \left.\times \mathrm{d} t_{r-1} \mathrm{~d} t_{r-2} \ldots \mathrm{d} t_{1}\right\}^{1 / p} \mathrm{~d} x \\
& \leqslant C t^{(r-1) / q}\left\{\int_{-h}^{h} \int_{-h}^{h} \ldots \int_{-h}^{h}\left|w\left(x+t_{1}+\ldots+t_{r-1}\right) \int_{x-h \Phi_{t}(x) / 2+t_{1}+\ldots+t_{r-1}}^{x+h \Phi_{t}(x) / 2+t_{1}+\ldots+t_{r-1}} \mathrm{~d} f^{(r-1)}(y)\right|^{p}\right. \\
& \left.\times \mathrm{d} t_{r-1} \mathrm{~d} t_{r-2} \ldots \mathrm{d} t_{1}\right\}^{1 / p} \mathrm{~d} x \\
& \leqslant C t^{(r-1) / q} \int_{|x| \leqslant \sigma(2 t)}\left\{\int_{-h}^{h} \int_{-h}^{h} \ldots \int_{-h}^{h}\left|\int_{x-h \Phi_{t}(x) / 2+t_{1}+\ldots+t_{r-1}}^{x+h \Phi_{t}(x) / 2+t_{1}+\ldots+t_{r-1}} w(y) \mathrm{d} f^{(r-1)}(y)\right|^{p}\right. \\
& \left.\times \mathrm{d} t_{r-1} \mathrm{~d} t_{r-2} \ldots \mathrm{d} t_{1}\right\}^{1 / p} \mathrm{~d} x \\
& \leqslant C t^{(r-1) / q} \int_{|x| \leqslant \sigma(2 t)}\left\{\int_{-h}^{h} \int_{-h}^{h} \ldots \int_{-h}^{h}\left(\int_{x-h \Phi_{t}(x) / 2+t_{1}+\ldots+t_{r-1}}^{x+h \Phi_{t}(x) / 2+t_{1}+\ldots+t_{r-1}} w(y)\left|\mathrm{d} f^{(r-1)}(y)\right|\right)^{p}\right. \\
& \left.\times \mathrm{d} t_{r-1} \mathrm{~d} t_{r-2} \ldots \mathrm{d} t_{1}\right\}^{1 / p} \\
& \leqslant C t^{(r-1) / q} \int_{|x| \leqslant \sigma(2 t)}\left(\int_{x-r h}^{x+r h} w(y)\left|\mathrm{d} f^{(r-1)}(y)\right|\right) \mathrm{d} x\left\{\int_{-h}^{h} \int_{-h}^{h} \ldots \int_{-h}^{h} \mathrm{~d} t_{r-1} \mathrm{~d} t_{r-2} \ldots \mathrm{d} t_{1}\right\}^{1 / p} \\
& \leqslant C t^{(r-1) / q} t^{(r-1) / p} \int_{|x| \leqslant \sigma(2 t)}\left(\int_{x-r h}^{x+r h} w(y)\left|\mathrm{d} f^{(r-1)}(y)\right|\right) \mathrm{d} x \\
& \leqslant C t^{r-1} \int_{|x| \leqslant \sigma(2 t)}\left(\int_{x-r h}^{x+r h} w(y)\left|\mathrm{d} f^{(r-1)}(y)\right|\right) \mathrm{d} x .
\end{aligned}
$$

Here we see

$$
\begin{aligned}
& \left.\int_{|x| \leqslant \sigma(2 t)}\left(\int_{x-r h}^{x+r h} w(y)\left|\mathrm{d} f^{(r-1)}(y)\right|\right) \mathrm{d} x \leqslant \int_{\mathbb{R}} w(y)\left(\int_{y-r h}^{y+r h} \mathrm{~d} x\right)\left|\mathrm{d} f^{(r-1)}(y)\right|\right) \\
& \left.\leqslant 2 r t \int_{\mathbb{R}} w(y)\left|\mathrm{d} f^{(r-1)}(y)\right|\right) .
\end{aligned}
$$

Hence we have

$$
\left\|w \Delta_{h \Phi_{t}(x)}^{r}(f, x)\right\|_{L^{1}(|x| \leqslant \sigma(2 t))} \leqslant C t^{r} \int_{\mathbb{R}} w(y)\left|\mathrm{d} f^{(r-1)}(y)\right| .
$$

By Theorem 9 we have

$$
\int_{\sigma(4 t)}^{\infty}\left|\left(Q^{\prime}(x)\right)^{r-1} w(x)\left(f(x)-P_{r-1, f}(x)\right)\right| \mathrm{d} x \leqslant\left\|w\left(f^{(r-1)}-c_{r-1, f}\right)\right\|_{L^{1}(|x| \geqslant \sigma(4 t))},
$$


where $c_{r-1, f}=P_{r-1, f}^{(r-1)}(x)$ is a constant. Now we see

$$
Q^{\prime}(\sigma(4 t)) \geqslant \frac{C}{t}
$$

In fact, we write $4 t=a_{u} / u$, then $\sigma(4 t)=a_{u}$. Hence we have

$$
Q^{\prime}(\sigma(4 t))=Q^{\prime}\left(a_{u}\right) \geqslant C_{1} \frac{u \sqrt{T\left(a_{u}\right)}}{a_{u}} \geqslant \frac{C_{1}}{4 t}
$$

that is, we have (A.2). Therefore,

$$
\begin{aligned}
& \int_{\sigma(4 t)}^{\infty}\left|w(x)\left(f(x)-P_{r-1, f}(x)\right)\right| \mathrm{d} x \\
& \leqslant C \frac{1}{\left(Q^{\prime}(\sigma(4 t))\right)^{r-1}}\left[\int_{\sigma(4 t)}^{\infty}\left|w(x)\left(f^{(r-1)}(x)-f^{(r-1)}(\sigma(4 t))\right)\right| \mathrm{d} x\right. \\
&\left.+\int_{\sigma(4 t)}^{\infty}\left|w(x)\left(f^{(r-1)}(\sigma(4 t))-c_{r-1, f}\right)\right| \mathrm{d} x\right] \\
& \leqslant C \frac{1}{\left(Q^{\prime}(\sigma(4 t))\right)^{r-1}}\left[\int_{\sigma(4 t)}^{\infty}\left|w(x) \int_{0}^{x} \mathrm{~d} f^{(r-1)}(y)\right| \mathrm{d} x\right. \\
&\left.+\int_{\sigma(4 t)}^{\infty}\left|w(x)\left(f^{(r-1)}(\sigma(4 t))-c_{r-1, f}\right)\right| \mathrm{d} x\right] \\
& \leqslant C \frac{1}{\left(Q^{\prime}(\sigma(4 t))\right)^{r}}\left[\int_{\sigma(4 t)}^{\infty} Q^{\prime}(x) w(x) \int_{0}^{x}\left|\mathrm{~d} f^{(r-1)}(y)\right| \mathrm{d} x\right. \\
&\left.+\left|f^{(r-1)}(\sigma(4 t))-c_{r-1, f}\right| \int_{\sigma(4 t)}^{\infty} Q^{\prime}(x) w(x) \mathrm{d} x\right] \\
& \leqslant C \frac{1}{\left(Q^{\prime}(\sigma(4 t))\right)^{r}}\left[\int_{\sigma(4 t)}^{\infty} Q^{\prime}(x) w(x) \int_{0}^{x}\left|\mathrm{~d} f^{(r-1)}(y)\right| \mathrm{d} x+\left|f^{(r-1)}(\sigma(4 t))-c_{r-1, f}\right| w(\sigma(4 t))\right] \\
& \leqslant C t^{r}\left[\int_{0}^{\infty} Q^{\prime}(x) w(x)\left(\int_{0}^{x}\left|\mathrm{~d} f^{(r-1)}(y)\right|\right) \mathrm{d} x+\left|f^{(r-1)}(\sigma(4 t))-c_{r-1, f}\right| w(\sigma(4 t)]\right. \\
& \leqslant C t^{r}\left[\int_{0}^{\infty}\left(\int_{y}^{\infty} Q_{0}^{\prime}(x) w(x) \mathrm{d} x\right)\left|\mathrm{d} f^{(r-1)}(y)\right|+\left|f^{(r-1)}(\sigma(4 t))-c_{r-1, f}\right| w(\sigma(4 t)]\right. \\
& \leqslant C t^{r}\left[\int_{0}^{\infty} w(y)\left|\mathrm{d} f^{(r-1)}(y)\right|+\left|f^{(r-1)}(\sigma(4 t))-c_{r-1, f}\right| w(\sigma(4 t))\right] \\
& \\
& \leqslant
\end{aligned}
$$

for $t$ small enough. Similarly,

$$
\int_{-\infty}^{-\sigma(4 t)}\left|w(x)\left(f(x)-P_{r-1, f}(x)\right)\right| \mathrm{d} x \leqslant C t^{r} \int_{-\infty}^{0} w(y)\left|\mathrm{d} f^{(r-1)}(y)\right| .
$$

Therefore, we have the result for $\tilde{\omega}_{r, 1}(w, f, t)$. 
Next we give the estimate for $\tilde{\omega}_{r, \infty}(w, f, t)$. Using the calculation of the proof of the above $L_{1}$ case, we have for any $\varepsilon>0$ there exists $L>0$ large enough such that

$$
\begin{aligned}
\| & w \Delta_{h \Phi_{t}(x)}^{r}(f, x) \|_{L^{\infty}(L \leqslant|x| \leqslant \sigma(2 t))} \\
\leqslant & C\left\{\int_{-h}^{h} 1^{q} \mathrm{~d} t_{r-1}\right\}^{1 / q}\left\{\int_{-h}^{h} 1^{q} \mathrm{~d} t_{r-2}\right\}^{1 / q} \ldots .\left\{\int_{-h}^{h} 1^{q} \mathrm{~d} t_{1}\right\}^{1 / q} \\
& \times\left\{\int_{-h}^{h} \int_{-h}^{h} \ldots \int_{-h}^{h} \sup _{L \leqslant|x|}\left|w\left(x+t_{1}+\ldots+t_{r-1}\right) g_{r-1}\left(x+t_{1}+\ldots+t_{r-1}\right)\right|^{p}\right. \\
& \left.\times \mathrm{d} t_{r-1} \mathrm{~d} t_{r-2} \ldots \mathrm{d} t_{1}\right\}^{1 / p} \mathrm{~d} x \\
\leqslant & C \varepsilon t^{(r-1) / q}\left\{\int_{-h}^{h} \int_{-h}^{h} \ldots \int_{-h}^{h} \mathrm{~d} t_{r-1} \mathrm{~d} t_{r-2} \ldots \mathrm{d} t_{1}\right\}^{1 / p} \mathrm{~d} x \\
\leqslant & C \varepsilon t^{r-1},
\end{aligned}
$$

and

$$
\begin{aligned}
& \left\|w \Delta_{h \Phi_{t}(x)}^{r}(f, x)\right\|_{L^{\infty}(|x| \leqslant L)} \leqslant C t^{r-1} \sup _{|x| \leqslant L} \int_{|x| \leqslant \sigma(2 t)}\left(\int_{x-r h}^{x+r h} w(y)\left|\mathrm{d} f^{(r-1)}(y)\right|\right) \mathrm{d} x \\
& \leqslant C \varepsilon t^{r-1}
\end{aligned}
$$

On the other hand, from Theorem 9 we easily see

$$
\left|\left(Q^{\prime}(x)\right)^{r-1} w(x)\left(f(x)-P_{r-1, f}(x)\right)\right| \leqslant C\left\|w\left(f^{(r-1)}(x)-c_{r-1, f}\right)\right\|_{L^{\infty}(|x| \geqslant \sigma(4 t))} .
$$

Hence, when $|x| \geqslant \sigma(4 t)$,

$$
\begin{aligned}
& \left|w(x)\left(f(x)-P_{r-1, f}(x)\right)\right| \leqslant C \frac{1}{\left(Q^{\prime}(\sigma(4 t))\right)^{r-1}}\left\|w\left(f^{(r-1)}-c_{r-1, f}\right)\right\|_{L^{\infty}(|x| \geqslant \sigma(4 t))} \\
& =C t^{r-1} o(1), \quad \text { as } t \rightarrow 0 .
\end{aligned}
$$

Here we use the fact that under the condition of (1.8) we see $\lim _{|x| \rightarrow \infty} w(x)\left(f^{(r-1)}(x)-c_{r-1, f}\right)=0$ (see Lemma 10). Therefore, we have the result.

\section{Proofs of Theorem 14 and Corollaries 15 and 16}

To prove Theorem 14 we need the following lemma.

Lemma 21 (cf. [12, Proposition 3.3]). Let $1 \leqslant p \leqslant \infty$ and $w \in \mathcal{F}_{\lambda}\left(C^{3}+\right)(0<\lambda<3 / 2)$ satisfy (1.2). Then for every absolutely continuous $g$ with $w^{\prime} \in L^{p}(\mathbb{R})$ and for any $n \in \mathbb{N}$, there exists a polynomial $V_{n} \in \mathcal{P}_{2 n}$ such that $V_{n}^{\prime}=v_{n}\left(g^{\prime}\right)$ and

$$
\left\|w\left(g-V_{n}\right)\right\|_{L^{p}(\mathbb{R})} \leqslant C E_{p, n}\left(T^{1 / 4} w, g^{\prime}\right)
$$

holds, where $v_{n}\left(g^{\prime}\right)$ is the de la Vallée Poussin mean of $g^{\prime}$.

Proof. We only repeat the proof of [12, Proposition 3.3]. We need, however,

$$
\left\|w\left(g^{\prime}-v_{n}\left(g^{\prime}\right)\right)\right\|_{L^{p}(\mathbb{R})} \leqslant C E_{p, n}\left(T^{1 / 4} w, g^{\prime}\right)
$$

instead of $[12,(3.12)$ in the proof of Proposition 3.3], that is,

$$
\left\|w\left(g^{\prime}-v_{n}\left(g^{\prime}\right)\right)\right\|_{L^{p}(\mathbb{R})} \leqslant C T^{1 / 4}\left(a_{n}\right) E_{p, n}\left(w, g^{\prime}\right) .
$$

In fact, (A.1) follows from Result 1 (1.4). 
$\left[A_{4}\right]$ (Proof of Theorem 14). We find a polynomial $V_{n-1}$ as in Lemma 21, so that $V_{n-1}^{\prime}=v_{n-1}\left(f^{\prime}\right)$. Then, from Result 1 (1.3) and Proposition 19 we get

$$
\begin{aligned}
& \left\|\frac{w}{T^{1 / 2}}\left(f^{\prime}-P^{\prime}\right)\right\|_{L^{p}(\mathbb{R})} \\
& \leqslant\left\|\frac{w}{T^{1 / 2}}\left(f^{\prime}-v_{n-1}\left(f^{\prime}\right)\right)\right\|_{L^{p}(\mathbb{R})}+\left\|\frac{w}{T^{1 / 2}}\left(V_{n-1}^{\prime}-P^{\prime}\right)\right\|_{L^{p}(\mathbb{R})} \\
& \leqslant C\left\{E_{p, n-1}\left(f^{\prime}\right)+\frac{n}{a_{n}}\left\|w\left(V_{n-1}-P\right)\right\|_{L^{p}(\mathbb{R})}\right\} .
\end{aligned}
$$

Here, by Lemma 21 and (4.1) we see

$$
\begin{aligned}
& \left\|w\left(V_{n-1}-P\right)\right\|_{L^{p}(\mathbb{R})} \leqslant\left\|w\left(f-V_{n-1}\right)\right\|_{L^{p}(\mathbb{R})}+\|w(f-P)\|_{L^{p}(\mathbb{R})} \\
& \leqslant C \frac{a_{n}}{n} E_{p, n-1}\left(T^{1 / 4} w, f^{\prime}\right)+\varepsilon .
\end{aligned}
$$

Hence, for $i=1$ we have (4.2), that is,

$$
\left\|\frac{w}{T^{1 / 2}}\left(f^{\prime}-P^{\prime}\right)\right\|_{L^{p}(\mathbb{R})} \leqslant C E_{p, n-1}\left(T^{1 / 4} w, f^{\prime}\right)+\frac{n}{a_{n}} \varepsilon .
$$

If in (A.4) we replace $w$ and $\varepsilon$ with

$$
w \rightarrow w_{-1 / 2} \sim T^{-1 / 2} w \text { and } \varepsilon \rightarrow C E_{p, n-1}\left(T^{1 / 4} w, f^{\prime}\right)+\frac{n}{a_{n}} \varepsilon
$$

respectively (note Result 4), then

$$
\begin{aligned}
& \left\|\frac{w}{T}\left(f^{\prime \prime}-P^{\prime \prime}\right)\right\|_{L^{p}(\mathbb{R})} \leqslant C E_{p, n-2}\left(T^{-1 / 4} w, f^{\prime \prime}\right)+\frac{n-1}{a_{n-1}}\left(C E_{p, n-1}\left(T^{1 / 4} w, f^{\prime}\right)+\frac{n}{a_{n}} \varepsilon\right) \\
& \leqslant C\left\{E_{p, n-2}\left(T^{1 / 4} w, f^{\prime \prime}\right)+\left(\frac{n}{a_{n}}\right)^{2} \varepsilon\right\}
\end{aligned}
$$

that is, we have (4.2) with $i=2$. We may continue this method. So, inductively we have (4.2) with $i=1,2, \ldots, r$, that is,

$$
\left\|\frac{w}{T^{i / 2}}\left(f^{(i)}-P^{(i)}\right)\right\|_{L^{p}(\mathbb{R})} \leqslant C\left\{E_{p, n-i}\left(T^{1 / 4} w, f^{(i)}\right)+\left(\frac{n}{a_{n}}\right)^{i} \varepsilon\right\} .
$$

In particular, if

$$
\|w(f-P)\|_{L^{p}(\mathbb{R})} \leqslant C E_{p, n}\left(T^{1 / 4} w ; f\right)
$$

then by (4.2) and Result 5,

$$
\begin{aligned}
& \left\|\frac{w}{T^{i / 2}}\left(f^{(i)}-P^{(i)}\right)\right\|_{L^{p}(\mathbb{R})} \leqslant C E_{p, n-i}\left(T^{1 / 4} w, f^{(i)}\right)+C\left(\frac{n}{a_{n}}\right)^{i} E_{p, n}\left(T^{1 / 4} w ; f\right) \\
& \leqslant C E_{p, n-i}\left(T^{1 / 4} w, f^{(i)}\right)+C E_{p, n-i}\left(T^{1 / 4} w ; f^{(i)}\right) \\
& \leqslant C E_{p, n-i}\left(T^{1 / 4} w ; f^{(i)}\right) \leqslant C\left(\frac{a_{n}}{n}\right)^{r-i} E_{p, n-r}\left(T^{1 / 4} w ; f^{(r)}\right),
\end{aligned}
$$

that is, we have (4.3).

$\left[A_{5}\right]$ (Proof of Corollary 15). From (4.4) we have

$$
\left\|\frac{w}{T^{1 / 4}}(f-P)\right\|_{L^{p}(\mathbb{R})} \leqslant \varepsilon .
$$

Let $T^{-1 / 4} w \sim w^{*} \in \mathcal{F}\left(C^{2}+\right)$ (see Result 4). Then, applying Theorem 14 with $w^{*}$, we have (4.5), that is,

$$
\left\|\frac{w}{T^{(2 i+1) / 4}}\left(f^{(i)}-P^{(i)}\right)\right\|_{L^{p}(\mathbb{R})} \leqslant C\left\{E_{p, n-i}\left(w, f^{(i)}\right)+\left(\frac{n}{a_{n}}\right)^{i} \varepsilon\right\} .
$$


In particular, if

$$
\|w(f-P)\|_{L^{p}(\mathbb{R})} \leqslant C E_{p, n}(w ; f),
$$

then by (4.5) and Result 5,

$$
\begin{aligned}
& \left\|\frac{w}{T^{(2 i+1) / 4}}\left(f^{(i)}-P^{(i)}\right)\right\|_{L^{p}(\mathbb{R})} \leqslant C E_{p, n-i}\left(w, f^{(i)}\right)+C\left(\frac{n}{a_{n}}\right)^{i} E_{p, n}(w ; f) \\
& \leqslant C E_{p, n-i}\left(w, f^{(i)}\right) \leqslant C\left(\frac{a_{n}}{n}\right)^{r-i} E_{p, n-r}\left(w ; f^{(r)}\right),
\end{aligned}
$$

that is, we have (4.7).

$\left[A_{6}\right]$ (Proof of Corollary 16). Noting (4.8), we set

$$
\left\|T^{i / 2} w(f-P)\right\|_{L^{p}(\mathbb{R})}=\varepsilon_{i} \leqslant \varepsilon, \quad i=1,2, \ldots, r .
$$

By Result 4 there is a weight $w_{i}$ such as $T^{i / 2} w \sim w_{i} \in \mathcal{F}\left(C^{2}+\right)$. Now we apply Theorem 5 with $w_{i}$, then from (4.2) we have (4.9), that is,

$$
\left\|w\left(f^{(i)}-P^{(i)}\right)\right\|_{L^{p}(\mathbb{R})} \leqslant C\left\{E_{p, n-i}\left(T^{(2 i+1) / 4} w, f^{(i)}\right)+\left(\frac{n}{a_{n}}\right)^{i} \varepsilon_{i}\right\} .
$$

In particular, if (4.10) holds, then for $i=1,2, \ldots, r$,

$$
\left\|T^{i / 2} w(f-P)\right\|_{L^{p}(\mathbb{R})} \leqslant C E_{p, n}\left(T^{r / 2} w ; f\right),
$$

and by (4.9) and Result 5,

$$
\left\|w\left(f^{(i)}-P^{(i)}\right)\right\|_{L^{p}(\mathbb{R})} \leqslant C\left(\frac{a_{n}}{n}\right)^{r-i} E_{p, n-r}\left(T^{(2 r+1) / 4} w ; f^{(r)}\right),
$$

that is, we have (4.11).

To prove Theorem 18 we need some lemmas. We define

$$
\varphi_{u}(x)= \begin{cases}\frac{a_{u}}{u} \frac{1-\frac{|x|}{a_{2 u}}}{\sqrt{1-\frac{|x|}{a_{u}}+\delta_{u}}},|x| \leqslant a_{u} ; \quad \delta_{u}=\left\{u T\left(a_{u}\right)\right\}^{-2 / 3}, u>0 . \\ \varphi_{u}\left(a_{u}\right), \quad a_{u}<\mid\end{cases}
$$

Lemma 22 ([8, Lemma 3.4]). We have

$$
\frac{a_{n}}{n} \frac{1}{\sqrt{T(x)}} \varphi_{n}^{-1}(x) \leqslant C .
$$

We define $L_{p}$-type Christoffel function $\lambda_{n, p}(w ; x)$ by

$$
\lambda_{n, p}(w ; x):=\inf _{P \in \mathcal{P}_{n}} \int_{-\infty}^{\infty}|w(t) P(t)|^{p} \mathrm{~d} t /|P(x)|^{p} .
$$

Lemma 23 ([4, Theorem $9.3(\mathrm{c})])$. Let $w \in \mathcal{F}\left(C^{2}+\right)$. Let $0<p<\infty$.

(a) Let $L>0$. Then uniformly for $n \geqslant 1$ and $|x| \leqslant a_{n}\left(1+L \eta_{n}\right)$, we have

$$
\lambda_{n, p}(w ; x) \sim \varphi_{n}(x) w^{p}(x) .
$$

(b) Moreover, uniformly for $n \geqslant 1$ and $x \in \mathbb{R}$,

$$
\varphi_{n}(x) w^{p}(x) \leqslant C \lambda_{n, p}(w ; x) .
$$

Now the proof of the theorem is simple. 
$\left[A_{7}\right]$ Proof of Theorem 18. The equation (5.1) follows from [4, Theorem 10.3]. We show (5.2). By Result 4 we can replace $T^{\frac{1}{2}\left(\frac{1}{q}-\frac{1}{p}\right)}$ with $w_{\alpha / 2} \in \mathcal{F}\left(C^{2}+\right)$, where $\alpha:=\frac{1}{q}-\frac{1}{p}$. Let $1 \leqslant q<p$.

$$
\begin{aligned}
& \left\|T^{-\alpha / 2} w P\right\|_{L_{p}(\mathbb{R})}^{p}=\int_{-\infty}^{\infty}\left|T^{-\alpha / 2}(t) w(t) P(t)\right|^{p} \mathrm{~d} t \\
& =\int_{-\infty}^{\infty}\left|T^{-p \alpha / 2}(t) \| w(t) P(t)\right|^{p-q}|w(t) P(t)|^{q} \mathrm{~d} t \\
& \leqslant \int_{-\infty}^{\infty}\left|T^{-q \alpha / 2}(t) \| w(t) P(t)\right|^{p-q}|w(t) P(t)|^{q} \mathrm{~d} t \\
& =\int_{-\infty}^{\infty}\left|\frac{T^{-\alpha / 2}(t) w(t) P(t)}{T^{\frac{\alpha}{2} \frac{q}{p-q}(t)}}\right|^{p-q}|w(t) P(t)|^{q} \mathrm{~d} t \\
& \leqslant\left\|\frac{\left|T^{-\alpha / 2} w P\right|^{p}}{\sqrt{T}}\right\|_{L_{\infty}(\mathbf{R})}^{\frac{p-q}{p}}\|w P\|_{L_{q}(\mathbb{R})}^{q},
\end{aligned}
$$

because of $\alpha p q /(p-q)=1$. Here we use some numbers and functions with the weight $T^{-\alpha / 2} \sim w^{*}:=$ $w_{-\alpha / 2} \in \mathcal{F}\left(C^{2}+\right)$. And they are MRS-numbers $a_{n}^{*}$ and the functions $T^{*}(x), \varphi_{n}^{*}(x)$. We see $L_{p}-$ type Christoffel functions $\lambda_{n, p}\left(w^{*} ; x\right)$. By Lemmas 22 and 23 we have

$$
\begin{aligned}
& \frac{\left|T^{-\alpha / 2}(t) w(t) P(t)\right|^{p}}{\sqrt{\overline{T(t)}}} \leqslant C \frac{w^{*}(t)^{p}}{\sqrt{T^{*}(t)}} \lambda_{n, p}^{-1}\left(w^{*} ; t\right)\left\|w^{*} P\right\|_{L_{p}(\mathbb{R})}^{p} \\
& \left.\leqslant C \frac{1}{\sqrt{T^{*}(t)}} \varphi_{n}^{*-1}(t)\right)\left\|w^{*} P\right\|_{L_{p}(\mathbb{R})}^{p} \\
& \leqslant C\left(\frac{n}{a_{n}^{*}}\right)\left\|w^{*} P\right\|_{L_{p}(\mathbb{R})}^{p} \leqslant C\left(\frac{n}{a_{n}}\right)\left\|T^{-\alpha / 2} w P\right\|_{L_{p}(\mathbb{R})}^{p} .
\end{aligned}
$$

Substituting (A.6) into (A.5), we have

$$
\begin{aligned}
& \left.\left\|T^{-\alpha / 2} w P\right\|_{L_{p}(\mathbb{R})}^{p} \leqslant C\left\{\left(\frac{n}{a_{n}}\right)\left\|T^{-\alpha / 2} w P\right\|_{L_{p}(\mathbb{R})}^{p}\right\}\right\}^{\frac{p-q}{p}}\|w P\|_{L_{q}(\mathbb{R})}^{q} \\
& =C\left(\frac{n}{a_{n}}\right)^{\frac{p-q}{p}}\left\|T^{-\alpha / 2} w P\right\|_{L_{p}(\mathbb{R})}^{p-q}\|w P\|_{L_{q}(\mathbb{R})}^{q} .
\end{aligned}
$$

So

$$
\left\|T^{-\alpha / 2} w P\right\|_{L_{p}(\mathbb{R})}^{q} \leqslant C\left(\frac{n}{a_{n}}\right)^{\frac{p-q}{p}}\|w P\|_{L_{q}(\mathbb{R})}^{q}
$$

that is,

$$
\left\|T^{-\alpha / 2} w P\right\|_{L_{p}(\mathbb{R})} \leqslant C\left(\frac{n}{a_{n}}\right)^{\frac{1}{q}-\frac{1}{p}}\|w P\|_{L_{q}(\mathbb{R})} .
$$

Consequently, we have the result (5.2). 